\title{
Application of pulse compression techniques to broadband acoustic scattering by live individual zooplankton ${ }^{\text {a) }}$
}

\author{
Dezhang Chu and Timothy K. Stanton \\ Department of Applied Ocean Physics and Engineering, Woods Hole Oceanographic Institution, \\ Woods Hole, Massachusetts 02543-1053
}

(Received 30 July 1996; accepted for publication 15 January 1998)

\begin{abstract}
Distinct frequency dependencies of the acoustic backscattering by zooplankton of different anatomical groups have been observed in our previous studies [Chu et al., ICES J. Mar. Sci. 49, 97-106 (1992); Stanton et al., ICES J. Mar. Sci. 51, 505-512 (1994)]. Based mainly on the spectral information, scattering models have been proposed to describe the backscattering mechanisms of different zooplankton groups [Stanton et al., J. Acoust. Soc. Am. 103, 236-253 (1998b)]. In this paper, an in-depth study of pulse compression (PC) techniques is presented to characterize the temporal, spectral, and statistical signatures of the acoustic backscattering by zooplankton of different gross anatomical classes. Data collected from various sources are analyzed and the results are consistent with our acoustic models. From compressed pulse (CP) outputs for all three different zooplankton groups, two major arrivals from different parts of the animal body can be identified: a primary and a secondary arrival. (1) Shrimplike animals (Euphausiids and decapod shrimp; near broadside incidence only): the primary one is from the front interface (interface closest to the transducer) of the animal and the secondary arrival is from the back interface; (2) gas-bearing animals (Siphonophores): the primary arrival is from the gas inclusion and the secondary arrival is from the body tissue ("local acoustic center of mass"); and (3) elastic shelled animals (Gastropods): the primary one is from the front interface and the secondary arrival corresponds to the subsonic Lamb wave that circumnavigates the surface of the shell. Statistical analysis of these arrivals is used to successfully infer the size of the individual animals. In conjunction with different aspects of PC techniques explored in this paper, a concept of partial wave target strength (PWTS) is introduced to describe scattering by the different $\mathrm{CP}$ highlights. Furthermore, temporal gating of the CP output allows rejection of unwanted signals, improves the output signal-to-noise ratio (SNR) of the spectra of selected partial waves of interest, and provides a better understanding of the scattering mechanism of the animals. In addition, it is found that the averaged PWTS can be used to obtain a more quantitative scattering characterization for certain animals such as siphonophores.

(C) 1998 Acoustical Society of America. [S0001-4966(98)02105-5]
\end{abstract}

PACS numbers: 43.10.Ln, 43.60.Cg, 43.60.Gk, 43.30.Ft, 43.30.Sf [JLK]

\section{INTRODUCTION}

Zooplankton aggregations often contain a diverse collection of animals of different anatomical groups, species, and sizes. Because of this diversity as well as the strong dependence of acoustic scattering by individual zooplankton upon acoustic frequency, and geometrical and physical properties of the animals, it is difficult, if not impossible, to make accurate predictions of zooplankton biomass by using a single frequency sonar. Sophisticated technology and scattering models are required for accurate estimates of biomass. Sonar systems with two or more discrete frequencies have been applied successfully to biomass estimation (Holliday et al., 1989; Holliday and Pieper, 1995) and animal behavior estimation (Chu et al., 1993) of simple populations containing a single species. Laboratory and shipboard experiments involving a combination of narrow band and broadband transducers have been conducted (Chu et al., 1992; Stanton et al., 1993b, 1998a) and much progress has been achieved in char-

\footnotetext{
a) "Selected research articles" are ones chosen occasionally by the Editorin-Chief, that are judged (a) to have a subject of wide acoustical interest, and (b) to be written for understanding by broad acoustical readership.
}

acterizing the zooplankton scattering. The broadband signals used in these reported experiments were a linear frequencymodulated signal (a "chirp") that continuously covers an octave band of frequencies. A broadband scattering signal is extremely powerful in analyzing and characterizing the signatures of the scattered signal which, in turn, leads to an understanding of the inherent scattering mechanisms.

There are two major domains in which broadband signals can be analyzed: frequency domain and time domain. Our previous studies (Chu et al., 1992; Stanton et al., 1993a, 1993b, 1994a, 1994b, 1998b) mainly focused on the frequency or spectral analysis. There are many advantages of using spectral analysis, such as to automatically reject the noise outside the frequency band of interest (out-of-band noise). In addition to the spectral analysis, temporal analyses also provide useful information. One of the most important features of the time series analysis is that for a broadband signal, a higher time-domain resolution, $1 / B$, where $B$ is the bandwidth of the signal, can be obtained through various forms of signal processing. For a sufficiently broad bandwidth of the signal (or equivalently, a sufficiently short pulse length), the different parts of an individual animal can be 

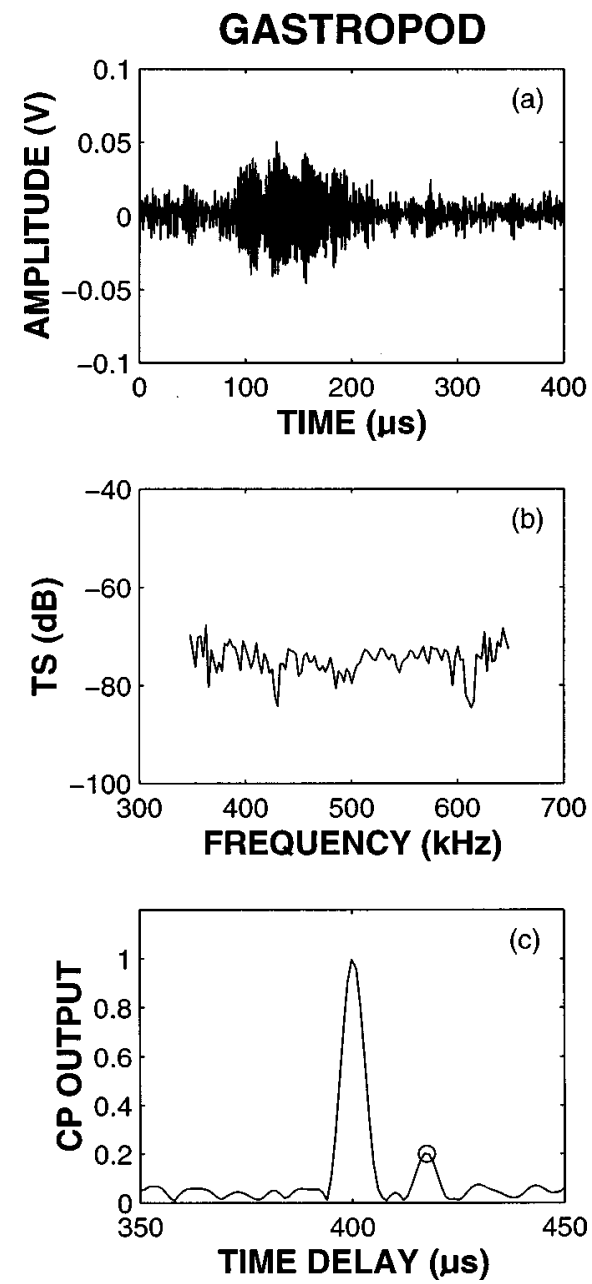

FIG. 1. Single ping backscattered signal from a 2-mm-long gastropod. (a) Time series; (b) target strength versus frequency; (c) compressed pulse (CP) output. The secondary arrival (circled peak) is clearly seen in (c) but its influence in the spectral domain is not noticeable in (b). The transmit signal is a chirp signal swept from $300 \mathrm{kHz}$ to $700 \mathrm{kHz}$ over $200 \mu \mathrm{s}$.

resolved acoustically. Ideally, in a noise-free environment, i.e., the signal-to-noise ratio (SNR) approaches infinity, the acoustic impulse response of the target can be obtained via either direct deconvolution (in the time domain) or the Fourier transform/Inverse Fourier transform process (in the frequency domain).

A combination of electrical noise of the data acquisition system and ambient noise in the water detected by the receiver degrades the quality of the data. Increasing the transmit power can help offset these effects but that improvement is restricted by the limitations of the power amplifier and transmit transducers. For a constant transmit power, the wider the bandwidth of the transmitter, the weaker the transmitted power spectral density, and hence the lower the SNR in the spectral domain at the receiver. In zooplankton applications, especially when an individual animal is involved, the received signal could be very noisy. As an example, the scattered signal by a 2-mm-long gastropod (Limacina retroversa, an elastic shelled animal) is shown in Fig. 1. Figure 1(a) is the time series of the received backscattering signal for a single ping and Fig. 1(b) is its power spectrum. The transmit signal is a linear frequency modulated signal (chirp) swept from $300 \mathrm{kHz}$ to $700 \mathrm{kHz}$ over $200 \mu$ s. Noise is quite apparent in the raw time series and results in some of the "hashness" in the power spectrum.

It is well known that when a long-wideband signal is used, i.e., $B T \gg 1$, where $B$ is the bandwidth and $T$ is the pulse length, the output SNR can be increased by applying pulse compression (PC) techniques. With this process, the length of the original signal is reduced which provides improved temporal resolution. One pulse compression technique, the matched filter (MF), maximizes the output SNR by cross-correlating the received signal with a noise-less replicate of the transmit signal. Figure 1(c) illustrates the compressed pulse $(\mathrm{CP})$ output after cross-correlating the received raw backscattering time series in Fig. 1(a) with the applied transmit signal (details of this analysis are given in Sec. II B). Here the output SNR and temporal resolution are clearly improved and two distinct arrivals can be identified. The primary arrival (largest) corresponds to the specular component of the backscattering from the front interface of the animal and the secondary arrival (circled peak) corresponds to a subsonic Lamb wave that circumnavigates the surface of the shell and sheds back to the reciver (Stanton et al., 1998b). Had this been a true MF, the output would have been a sinc-like function. Since the scattering characteristics of the target were not incorporated into the processing, the actual output departs form the idealized MF output (i.e., resulting in multiple highlights). This deviation contains information regarding the scattering properties of the target.

Given the potential advantages of using PC techniques with broadband scattered signals by zooplankton, we present in this paper a study of the performance of PC processing in the context of acoustic scattering by zooplankton with the purpose of extracting more useful information acoustically. Since MF processing is, in part, a basis for this analysis, the background of MF's is given briefly in Sec. I. In Sec. II, different theoretical considerations of PC processing involving zooplankton scattering are studied. CP outputs of the backscattering data from different zooplankton groups collected from various sources are presented and analyzed in Sec. III, and finally, the conclusions are drawn in Sec. IV in which the advantages of using PC techniques in the application of zooplankton scattering are summarized.

\section{BACKGROUND OF MATCHED FILTER PROCESSING}

Matched filters are widely used in radar and sonar applications (Price, 1956; Siebert, 1956; Parvulescu, 1961; Cook and Bernfeld, 1967; Clay, 1987; Thorne et al., 1994, 1995), and their theoretical background can be found in many references (Van Vleck and Middleton, 1946; Turin, 1960; Van Trees, 1968; Whalen, 1971; Robinson, 1980; Winder and Loda, 1981). In this section, only a brief review of the theory and its application to the class of signals used in our experiments will be presented.

Matched filters are designed to maximize the output SNR for a given input SNR when noise is present. Assume that a time series $x(t)$ is composed of two components: a signal $s(t)$ and noise $n(t)$ [i.e., $x(t)=s(t)+n(t)$ ], and is fed into a filter whose impulse response is $a(t)$. The filtered output $y(t)$ can be expressed as 


$$
y(t)=x(t) * a(t)=s(t) * a(t)+n(t) * a(t),
$$

where the symbol " $*$ "' denotes convolution. The ratio of the instantaneous power of the signal to that of the noise at time $\tau$ is

$$
\Gamma(\tau)=\frac{\left(\int_{0}^{\tau} a(t) s(\tau-t) d t\right)^{2}}{\left\langle\left(\int_{0}^{\tau} a(t) n(\tau-t) d t\right)^{2}\right\rangle} .
$$

Maximizing Eq. (2) with respect to the filter $a(t)$ results in (Whalen, 1971)

$$
\int_{0}^{\tau} a(\xi) r_{n}(t-\xi) d \xi=k_{c} s(\tau-t),
$$

where $r_{n}$ is the autocorrelation function of the random noise and $k_{c}$ is a normalization constant. If the noise is white, $r_{n}$ becomes a delta function, the above equation reduces to

$$
a(t)=k_{c} s(\tau-t) .
$$

Such a filter is called a matched filter since its coefficients are "matched" to the applied signal $s(t)$. Equation (4) shows that a MF is merely a time reversed sequence of the original signal. Since a convolution with a time reversed function is mathematically equivalent to the correlation with that function without time reversal, a MF is also referred to as a correlator. Substituting Eq. (4) into Eq. (1) and dropping the time shift $\tau$, we have

$$
\begin{aligned}
y(t) & =x(t) * s(-t) \\
& =k_{c} s(t) * s(-t)+k_{c} n(t) * s(-t) \\
& =k_{c} r_{s s}(t)+k_{c} s(t) \otimes n(t),
\end{aligned}
$$

where " $\otimes$ ' stands for correlation and $r_{s s}(t)$ is the autocorrelation function of the signal $s(t)$. For white noise, $n(t)$, the second term in Eq. (5) tends to zero. It can be proven that the time-domain resolution of a MF output is approximately equal to $1 / B$, and the processing gain - the ratio of output SNR to input SNR is proportional to $2 B T$, where $B$ is the bandwidth and $T$ is the pulse length of the applied signal (Turin, 1960).

One of the widely used signals to provide a high processing gain is a chirp signal because of its uniform coverage of frequencies within a given band. An ideal "up-sweep" chirp, a signal whose instantaneous frequency increases linearly with time, can be represented in the following form:

$$
u(t)=\left\{\begin{array}{l}
\cos \left(\omega_{0} t+\alpha t^{2}\right), \quad 0 \leqslant t \leqslant T, \\
0, \quad \text { otherwise, }
\end{array}\right.
$$

where $\omega_{0}, \alpha$, and $T$ are the initial angular frequency, sweep rate, and pulse length, respectively. It can be shown that for $\sqrt{\alpha} T \gg 1$, the analytical expression of the MF output of Eq. (6) is approximately

$$
R_{f}(\tau) \simeq \frac{1}{2} \cos \left[\left(\omega_{0}+\alpha T\right) \tau\right] \operatorname{Sinc}(\alpha \tau T),
$$

where $\tau$ is the time delay, $R_{f}(\tau)$ is the autocorrelation function of a continuous signal modulated by a rectangular window function and is defined in more general terms in Eq. (A2) of Appendix A. The Sinc function in Eq. (7) is defined as $\operatorname{Sinc}(x) \equiv \sin (x) / x$. The above approximate expression is based on the conditions that $\sqrt{\alpha} T \gg 1$ and $\tau \ll T$. The cosine

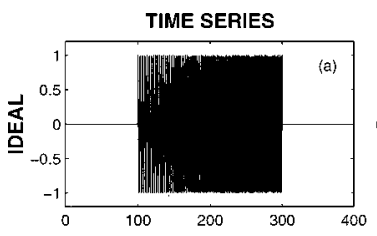

NORMALIZED MF OUTPUT
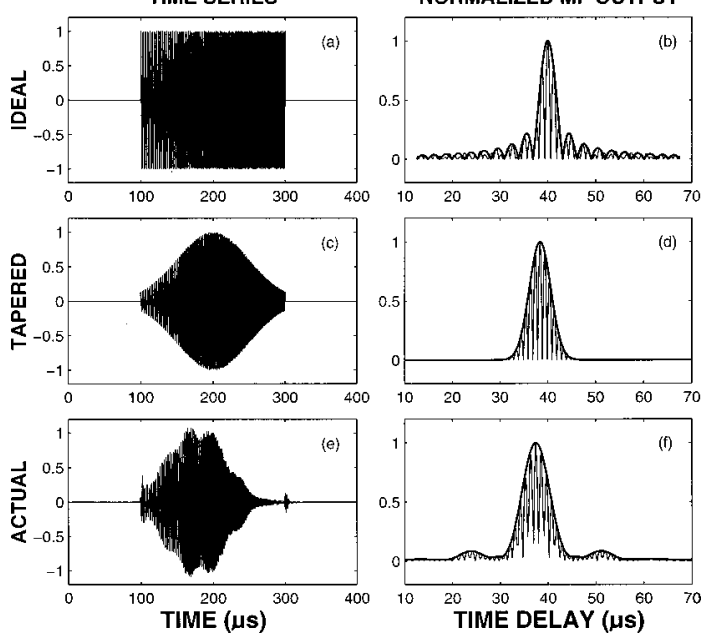

FIG. 2. Time series and MF outputs of various kinds of signals. (a), (b) Ideal chirp (300 kHz to $700 \mathrm{kHz}$ up sweep) with $f_{0}=300 \mathrm{kHz}, \alpha=2 \pi$ $\times 10^{9} \mathrm{~s}^{-2}$, and $T=200 \mu \mathrm{s}$. The envelope (thick line) of the MF output is computed from the Sinc function in Eq. (7). (c), (d) Gaussian-tapered chirp with $\beta=2 \times 10^{8} \mathrm{~s}^{-2}$ and the other parameters are the same as in (a). The envelope of the MF output (thick line) is computed from Eq. (10). (e), (f) Actually measured chirp signal received in the calibration mode illustrating the system response to the transmit signal given in (a). In each plot in the right column, the thick solid line is the demodulated MF output and the thin line is the full rectified MF output before demodulation.

term acts as a carrier signal whose angular frequency is the center angular frequency of the original chirp, $\left(\omega_{0}+\alpha T\right)$, while the term involving the Sinc function is the envelope of the MF output. An example of the time series $u(t)$ and its corresponding MF output are shown in Fig. 2(a) and (b), where $f_{0}=300 \mathrm{kHz}, \quad \alpha=2 \pi \times 10^{9} \mathrm{~s}^{-2}, \quad$ and $T=200 \mu \mathrm{s}$ (these parameters are sometimes used in our zooplankton scattering experiments). The resultant bandwidth is about $400 \mathrm{kHz}$. The envelope of the MF output is computed from the Sinc function in Eq. (7) and plotted with the thick solid line. Strong sidelobes are observed in Fig. 2(b) which are due to the sharp edges of the signal.

In reality, due to the nonuniform band-limited frequency response of most transducers such as the ones used in our experiments, the transmitted signal is typically tapered on its rising and falling edges. Such a signal can be reasonably approximated by an untapered chirp signal given by Eq. (6) modulated by a Gaussian envelope symmetric about $t=T / 2$

$$
u_{g}(t)=u(t) e^{-\beta(t-T / 2)^{2}},
$$

where $\beta$ is a constant that controls the degree of tapering. For $\sqrt{\beta} T \gg 1$, the analytical expression of its MF output becomes (Appendix A)

$$
R_{f}(\tau)=\frac{1}{4 T} \sqrt{\frac{2 \pi}{\beta}} e^{-\left[\left(\beta^{2}+\alpha^{2}\right) / 2 \beta\right] \tau^{2}} \cos \left[\left(\omega_{0}+\alpha T\right) \tau\right]
$$

The limiting condition of $\sqrt{\beta} T \gg 1$ corresponds to the case when the leading and trailing edges of the chirp are negligibly small at the beginning and end of the window function, respectively; that is, the windowed signal has the appearance of varying smoothly in time. The MF output in 
Eq. (9) has the same carrier signal as the untapered chirp case but with a Gaussian envelope given by

$$
R_{\text {env }}(\tau)=\frac{1}{4 T} \sqrt{\frac{2 \pi}{\beta}} e^{-\left[\left(\beta^{2}+\alpha^{2}\right) / 2 \beta\right] \tau^{2}} .
$$

Figure 2(c) and (d) shows this Gaussian tapered chirp time series and its MF output with a tapering coefficient $\beta$ $=2 \times 10^{8} \mathrm{~s}^{-2}$, where the thick line is the envelope computed using Eq. (10). For the purpose of comparison, the actual received transmit signal used during our 1994 cruise and its MF output are shown in Fig. 2(e) and (f). The modulation of its amplitude is due to the frequency response of the combination of the acoustic transducers and the data acquisition system described in Stanton et al. (1998a). The thick solid line is the envelope of the MF output (i.e., demodulated MF output). As expected, the Gaussian modulation widens the main lobe and reduces the sidelobe levels of the MF output [Fig. 2(d) and (f)]. Comparison of the time series and the MF output of the Gaussian tapered chirp with those of the actual received signal indicates that the Gaussian tapered chirp describes the actual received signal reasonably well.

\section{BASIC CHARACTERISTICS OF PULSE-COMPRESSED SCATTERED SIGNALS}

Since the scattering impulse response of a target is typically unknown, it is, in general, impossible to determine a real "replicate" of the received scattered signal and hence the signal will never be truly "matched" as in the case described above for MF processing. However, deviations of the received scattered signal from the expected output for the idealized (matched) case, can provide useful information about the target after processing. For example, if the idealized replicate is constructed assuming that the target is a point scatterer but the target is in fact of finite size, then the CP output will consist of multiple arrivals or "partial waves" from the target. This is in contrast to the expected single return from a point scatterer. The separations between the multiple arrivals may be related to the size of the target. In addition to the effects due to a finite body, there will be multiple arrivals due to a collection of multiple targets which will also affect the performance of the PC processing. While the mathematical treatment of both cases is similar, we will focus on the different characteristics of the CP output when the scattering from a single target is involved. Special attention will be focussed on relating the physics of the scattering to the deviations.

In many signal detection applications (Van Vleck and Middleton, 1946; Price, 1956; Siebert, 1956; Parvulescu, 1961), the absolute level and the shape of the CP output may not necessarily be as important as the accuracy of the arrival times of the detected echoes and their relative levels. However, to characterize the acoustic scattering by zooplankton of different groups, not only is the timing important but also the absolute level of the scattering, such as the target strength (TS). To determine the TS from a CP output of a scattering signal, special care must be taken. In the following part of this section, the basic scattering equations are presented and then incorporated into PC processing.

\section{A. Basic scattering equations}

For a backscattering geometry, ignoring the attenuation in water, the far field received pressure time series $p_{\mathrm{bs}}(t)$ for an individual target due to a point source is

$$
p_{\mathrm{bs}}(t)=\frac{s_{0}\left(t-t_{0}\right)}{r^{2}} * f_{\mathrm{bs}}(t),
$$

where $t_{0}=2 r / c, r$ is the distance between the transmitter (receiver) and the target, and $c$ is the sound speed in water. $s_{0}(t)$ is the source function and $f_{\mathrm{bs}}(t)$ is the backscattering impulse response of the target. Its Fourier transform $P_{\mathrm{bs}}(f)$ can be expressed in terms of the backscattering amplitude $F_{\text {bs }}$, which is the Fourier transform of the scattering impulse response $f_{\text {bs }}$, as,

$$
P_{\mathrm{bs}}(f)=S_{0}(f) \frac{e^{2 i k r}}{r^{2}} P_{\mathrm{bs}}(f)=P_{0}(f) \frac{e^{i k r}}{r} F_{\mathrm{bs}}(f),
$$

where $S_{0}$ is the Fourier transform of the source function $s_{0}$, and $k$ is the wave number of the transmit signal. $P_{0}$ $=S_{0} e^{i k r} / r$ is the incident wave at the target. For far field applications, $P_{0}$ can be treated as a plane incident wave. Notations of lower case and upper case are chosen to be consistent with the convention for time/frequency Fourier pairs. Here $F_{\text {bs }}$ corresponds to the backscattering amplitude $f_{\text {bs }}$ in our previous papers involving scattering models (e.g., Stanton et al., 1993a, 1993b, 1998b).

Target strength is defined in terms of the differential backscattering cross section $\sigma_{\text {bs }}$ and the backscattering amplitude $F_{\text {bs }}$ as (Clay and Medwin, 1977)

$$
\mathrm{TS}=10 \log _{10} \sigma_{\mathrm{bs}}=20 \log _{10}\left|F_{\mathrm{bs}}\right| \quad \mathrm{dB} \text { re: } 1 \mathrm{~m}^{2} .
$$

The scattering amplitude $F_{\text {bs }}$ is a measure of the scattering ability of a target at a range of 1 meter subject to a plane incident wave and can be expressed in terms of directly measurable quantities of transmit and received voltages as

$$
F_{\mathrm{bs}}=\frac{V_{\mathrm{bs}}^{(r)}}{V_{\mathrm{cal}}^{(r)}} \frac{V_{\mathrm{cal}}^{(t)}}{V_{\mathrm{bs}}^{(t)}} \frac{r_{\mathrm{bs}}^{2}}{r_{\mathrm{cal}}},
$$

where $V$ is the Fourier transform of the voltage, $r_{\mathrm{bs}}$ is the distance between the transducers and the target in backscattering mode, and $r_{\text {cal }}$ is the distance between the transmitter and the receiver in bistatic calibration mode, superscripts $t$ and $r$ stand for transmit and receive, respectively, and subscripts bs and cal stand for backscattering mode and calibration mode, respectively. In the backscattering mode, two closely spaced transducers are used, one for transmission and the other for reception. In the calibration mode, the same two transducers are separated by a distance of $r_{\text {cal }}$ and facing each other (Stanton et al., 1998a).

To accurately estimate the frequency dependent TS, the characteristics of the compressed pulse in both time and frequency domains need to be studied.

\section{B. Time domain-Cross correlation}

As mentioned above, if the received signal $s(t)$ in Eq. (1) is the scattered signal from a target, it is no longer an exact replica of the transmitted signal $s_{0}(t)$, but a convolution of $s_{0}(t)$ with the scattering impulse response of the tar- 
get $f_{\mathrm{bs}}(t)$ plus a noise component $n(t)$. Since the scattering impulse response is a complicated function of the geometric and physical properties of the target, it is not practical to find a function that truly matches the received scattering signal $f_{\mathrm{bs}} * s_{0}$ as a replica required by MF processing. An alternative candidate is the transmit signal. Since the transmit signal does not truly match the received scattering signal, to distinguish between the true MF processing and the processing using the transmit signal as the replica, we call such a nonideal MF processing pulse compression (PC) processing and its output compressed pulse (CP) output.

The resultant output in this scattering case can then be expressed as

$$
\begin{aligned}
y(t) & \simeq k_{c} s_{0}(t) \otimes\left(\frac{1}{r^{2}} f_{\mathrm{bs}}(t) * s_{0}\left(t-t_{0}\right)+n(t)\right), \\
& =\frac{k_{c}}{r^{2}} r_{s s}\left(t-t_{0}\right) \otimes f_{\mathrm{bs}}(t)+k_{c} r_{s n}(\tau),
\end{aligned}
$$

where $k_{c}$ is a proportionality constant, $\tau$ is time delay, $r_{s s}$ is the autocorrelation function of $s_{0}(t), r_{s n}$ is the crosscorrelation function of $s_{0}(t)$ and $n(t)$, and $t_{0}$ is defined in Eq. (11). In obtaining Eq. (16) from Eq. (15), we have used the results of Eq. (B7) in Appendix B. For white noise that is of sufficiently low level, the second term on the right hand side is small compared with the first term and Eq. (16) can be written approximately as

$$
y(\tau)=\frac{k_{c}}{r^{2}} r_{s s}\left(t-t_{0}\right) \otimes f_{\mathrm{bs}}(t) .
$$

For the special case where the scattering impulse response is a delta function $f_{\mathrm{bs}}(t)=\delta(t)$ (which corresponds to a point scatterer of uniform response), the resultant $\mathrm{CP}$ output $y(\tau)$ will be a simple product of a scaling constant and the autocorrelation function of the transmit signal. This ideal case represents true matched filtering.

If the scattering impulse response $f_{\mathrm{bs}}(t)$ is not a single impulse but involves a number of impulsive arrivals separated in time, then it can be written in terms of a sum of those arrivals (Ehrenbreg et al., 1978):

$$
\begin{aligned}
f_{\mathrm{bs}}(t) & =\sum_{j=1}^{N} f_{\mathrm{bs}}\left(t-\Delta_{j}\right), \\
& =\sum_{j=1}^{N} k_{s_{j}} \delta\left(t-\Delta_{j}\right),
\end{aligned}
$$

where the difference in spreading loss among the arrivals is ignored, $N$ is the total number of arrivals, and $\Delta_{j}$ is the difference of arrival time between the $j$ th arrival and the reference time $t_{0}=2 \mathrm{r} / \mathrm{c}$. For an applied chirp signal given in Eq. (6), the CP output of the scattering described above is a superposition of a series of Sinc-function-like arrivals with different amplitudes. If the separation time between arrivals is greater than $1 / B$, where $B$ is the band width of the chirp signal, these $N$ arrivals can be resolved in the time domain after processing. For a more general case where $f_{\mathrm{bs}_{j}}\left(t-\Delta_{j}\right)$ is an arbitrary function (Thorne et al., 1995), the CP output yields

$$
y(\tau)=\frac{k_{c}}{r^{2}} \sum_{j=1}^{N} r_{s s}\left(t-t_{0}\right) \otimes f_{\mathrm{bs}_{j}}\left(t-\Delta_{j}\right) .
$$

\section{Frequency domain-Spectrum average}

To further understand how PC processing can improve the output SNR, we express Eq. (16) as a summation of two integrals over frequency

$$
\begin{aligned}
y(\tau)= & y_{1}(\tau)+y_{2}(\tau), \\
= & \frac{k_{c}}{r^{2}} \int_{-\infty}^{\infty} F_{\mathrm{bs}}(f) R_{s s}(f) e^{i \omega\left(\tau-t_{0}\right)} d f \\
& +k_{c} \int_{-\infty}^{\infty} N(f) S_{0}(-f) e^{i \omega \tau} d f,
\end{aligned}
$$

where $F_{\mathrm{bs}}(f), R_{s s}(f), S_{0}(f)$, and $N(f)$ are the Fourier transforms of the backscattering impulse response $f_{\mathrm{bs}}(t)$, the autocorrelation function $r_{s s}(t)$, the transmit signal $s_{0}(t)$, and noise $n(t)$, respectively.

Clearly, the right-hand side of Eq. (20) is in the form of the inverse Fourier transform. By taking the Fourier transform of both sides of Eq. (20), the scattering amplitude $F_{\mathrm{bs}}$ can be obtained. However, directly taking the Fourier transform of $y(\tau)$ in Eq. (20) is equivalent to reversing the PC processing (Appendix C), and thus reduces the SNR back to that of the original signal. In other words, we cannot improve our estimate on the frequency dependent scattering amplitude $F_{\text {bs }}$.

However, evaluating the $\mathrm{CP}$ output $y(\tau)$ given by Eq. (20) at $\tau=t_{0}$, we obtain,

$$
y\left(t_{0}\right)=\frac{k_{c}}{r^{2}} \int_{-\infty}^{\infty} F_{\mathrm{bs}}(f) R_{s s}(f) d f,
$$

where the term associated with noise, $y_{2}(\tau)$, is neglected. Equation (21) is simply a weighted averaging process over a frequency band (band-limited case). Assuming $F_{\text {bs }}$ has a slowly varying phase over the frequency band of interest, and since the weighting function $R_{s s}(f)=S_{0}^{*} S_{0}$ is always a real function, all frequency components of the integrand are in phase and add up constructively resulting in an enhanced signal level. In contrast, for random noise described by the second term of Eq. (20), the phase of its Fourier components can be described as randomly and uniformly distributed over $\left[\begin{array}{ll}0 & 2 \pi\end{array}\right]$. Such random noise always mismatches the filter, hence all frequency components tend to add destructively resulting in a reduced noise level. It is the constructive addition for a matched signal and the destructive addition for random noise in the frequency domain that makes a PC processing improve the output SNR in the time domain.

\section{Single nonideal arrival}

As discussed in Sec. IB, the ideal case is when the scattering impulse response is a delta function, i.e., $f_{\mathrm{bs}}(t)$ $=k_{s} \delta(t)$, in the case of a point scatterer (the Fourier transform of the arrival is $\left.F_{\mathrm{bs}}(f)=k_{s}\right)$. The CP output is a simple integration of $R_{s s}$ over the entire frequency domain. In this case, the power density of the transmit signal is added constructively since the phase of the scattering transfer function 
$F_{\text {bs }}(f)$ is zero. However, in reality due to the finite size of any actual targets, the scattering impulse response $f_{\text {bs }}(t)$ will never be an ideal delta function, but a function that spreads out in the time domain and whose amplitude and phase spectra are functions of frequency. The $\mathrm{CP}$ output $y\left(t_{0}\right)$ of such a function is expected to be smaller than that due to a delta function having the same "strength" (i.e., the integration of the scattering impulse response $f_{\mathrm{bs}}(t)$ over the time it spans).

In this section, the influences of a nonideal scattering function on its CP output will be studied. The analysis can provide guidance in interpreting the results based on the $\mathrm{CP}$ outputs. A triangle function is chosen as the scattering impulse response since it can be easily changed from a delta function to a non-ideal impulse response with a finite bandwidth by simply varying the spread of the triangle function. Its strength is kept at unity; i.e., the area under the triangle is equal to 1 and the peak value at $t=0$ increases as the spread $\tau$ of the scattering impulse response decreases. For $\tau \longrightarrow 0$, the triangle function approaches a delta function with unit strength. Its Fourier transform can be expressed as (Bracewell, 1986):

$$
F_{\mathrm{bs}}(f)=\left(\frac{\sin (\omega \tau / 4)}{\omega \tau / 4}\right)^{2}
$$

where $\omega=2 \pi f$ is the angular frequency. To evaluate the performance of the $\mathrm{PC}$ processing due to the impulse response given by Eq. (22), a ratio function can be defined as,

$$
R_{f}(\tau)=20 \log _{10}\left(\frac{y_{f}\left(t_{0}\right)}{y_{d}\left(t_{0}\right)}\right)
$$

where $y_{d}\left(t_{0}\right)$ is the CP output at $\tau=t_{0}$ using a delta function as the scattering impulse response and $y_{f}\left(t_{0}\right)$ is the CP output at $\tau=t_{0}$ using a triangle impulse response. Figure 3 shows the dependence of $R_{f}$ upon the spread of the scattering impulse responses. For a scattering impulse response having spreads of $4 \mu \mathrm{s}$ and $8 \mu \mathrm{s}$, the $\mathrm{CP}$ output levels are reduced by $1.5 \mathrm{~dB}$ and $5 \mathrm{~dB}$, respectively, which indicates that for a scattered signal having the same strength but having a different spread, the output levels of a CP could differ by as much as several $\mathrm{dB}$.

As discussed before, $y\left(t_{0}\right)$ is a weighted average of the impulse response of the received backscattering signal over the frequency bandwidth. In some applications, we may be only interested in an averaged scattering level over a certain frequency band. For example, in Fig. 4 of Stanton et al. (1998a), an averaged TS over a frequency band from 400 to $500 \mathrm{kHz}$ is computed to characterize the scattering by an individual gastropod over that frequency band.

To analyze a particular band of interest, we integrate Eq. (21) within a specified frequency window resulting in a partial PC operation. To evaluate the performance of this partial PC processing, we define the ratio $R_{p}\left(f_{T}\right)$, which measures the deviation of the weighted average from its true average as:

$$
R_{p}\left(f_{T}\right)=20 \log _{10}\left|\int_{f_{0}-f_{T} / 2}^{f_{0}+f_{T} / 2} F_{\mathrm{bs}}(f) R_{s s}(f) d f / \Lambda\left(f_{T}\right)\right|,
$$

where $f_{0}$ and $f_{T}$ are the center frequency and the width of the frequency window, respectively, and $\Lambda\left(f_{T}\right)$ is a normalization factor defined as

$$
\Lambda\left(f_{T}\right)=\frac{1}{f_{T}} \int_{f_{0}-f_{T} / 2}^{f_{0}+f_{T} / 2} F_{\mathrm{bs}}(f) d f \int_{f_{0}-f_{T} / 2}^{f_{0}+f_{T} / 2} R_{s s}(f) d f
$$

It is apparent from Eq. (24) that if the scattering impulse response is a delta function, its Fourier transform $F_{\mathrm{bs}}(f)$ $=1$ and $R_{p}\left(f_{T}\right)$ is independent of the window width $f_{T}$. However, for a scattering impulse response that deviates from a delta function, $R_{p}\left(f_{T}\right)$ is no longer independent of $f_{T}$. Figure 4 illustrates the dependence of $R_{p}\left(f_{T}\right)$ on the width of the frequency window $f_{T}$, where the scattering impulse response is assumed to be a $10-\mu$ s triangle function and the frequency window is centered at $500 \mathrm{kHz}$. Three different transmit signals are used: (1) chirp without Gaussian tapering (dash-dotted); (2) chirp with Gaussian tapering (dashed); and (3) actually received signals in the calibration mode (solid). It can be seen that the deviation increases as the window width increases. For a window width less than $100 \mathrm{kHz}$, all three transmit signals have similar deviations less than $0.08 \mathrm{~dB}$, while for a $300-\mathrm{kHz}$ frequency window, there are deviations of about $2.2 \mathrm{~dB}$ for the Gaussian tapered and measured chirps and $0.2 \mathrm{~dB}$ for the untapered chirp. Again, we see that a Gaussian-tapered chirp gives similar results as the actual transmit signal.

Figure 4 suggests that the narrower the frequency window, the better the estimate of the average scattering response over that window. However, the narrower frequency window will degrade the performance of the PC, i.e., it reduces the output SNR. Figure 5 shows the influence of the frequency window on the SNR. The transmit signal is an actually measured calibration signal shown in Fig. 2(e). To compute the averaged output SNR from a windowed CP output, random noise is added to maintain the SNR at $11 \mathrm{~dB}$ before applying a PC.

The output SNR approaches a constant level (about 20 $\mathrm{dB}$ ) as the width of the frequency window increases. From Figs. 4 and 5, we can conclude that to improve the SNR, it is important to average over as wide a frequency band as possible. However, if a more accurate estimate of the scattering response at a particular frequency is desired, a narrower window PC processing is required. In other words, there is a trade-off in selecting the most appropriate window width.

\section{Multiple arrivals from a single target}

If the scattering function is a superposition of multiple arrivals separated in the time domain and given by Eq. (19), we rewrite Eq. (21) as,

$$
y(\tau)=\frac{1}{r^{2}} \sum_{j=1}^{N} k_{c j} \int_{-\infty}^{\infty} F_{\mathrm{bs}}^{(j)}(f) R_{s s}(f) e^{i \omega\left(t-t_{j}\right)} d f,
$$

where $t_{j}=2 r_{j} / c=t_{0}-\Delta_{j}, t_{0}$ and $\Delta_{j}$ are defined in Eqs. (11) and (18).

For a special case that $N=2$, we define a ratio function 


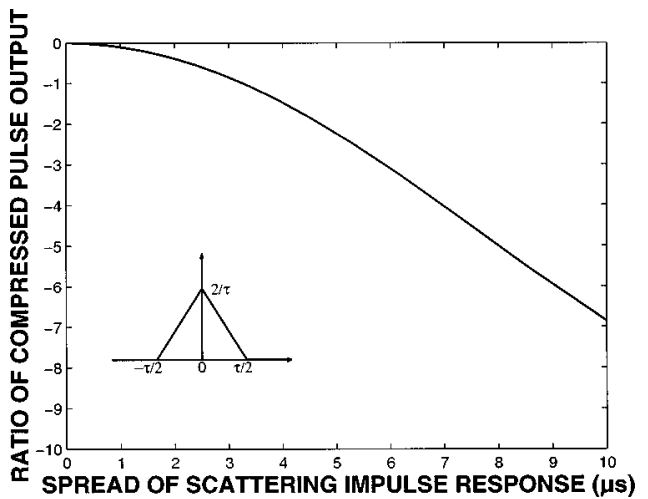

FIG. 3. Influence of the spread of a triangle scattering impulse response on the CP output. The transmit signal is an untapered chirp swept from 300 kHz to $700 \mathrm{kHz}$ over $200 \mu$ s shown in Fig. 2(a). The area of the scattering impulse response function is kept at unity.

$R_{T}(\Delta)=20 \log _{10}\left|\frac{\int_{-\infty}^{\infty} R_{s s}(f)\left(F_{\mathrm{bs}}^{(1)}(f)+F_{\mathrm{bs}}^{(2)}(f) e^{i \omega \Delta}\right) d f}{\int_{-\infty}^{\infty} R_{s s}(f) d f}\right|$,

where $\Delta=\delta_{2}-\delta_{1}$ is the time separation between the two arrivals. Figure 6 shows the influence of the separation between two arrivals of the same strength on the CP output. The transmit signal is an untapered chirp shown in Fig. 2(a), and the received backscattered signal is the superposition of two ideal delta functions having the same strength, i.e., $F_{\mathrm{bs}}^{(1)}=F_{\mathrm{bs}}^{(2)}=1$.

From Fig. 6, we find that $R_{T}(0)$ is $6 \mathrm{~dB}$ when the two arrivals coincide in time and tends to $0 \mathrm{~dB}$ as the separation tends to infinity. Note that for a separation between two arrivals greater than $1 / B=2.5 \mu \mathrm{s}$, the fluctuation of $R_{T}(\Delta)$ is less than $2 \mathrm{~dB}$. Figure 6 suggests that when the separation between two arrivals is less than the time domain resolution of the $\mathrm{CP}$, the echoes are not resolvable. In other words, a large output of the PC processing could result from a strong scattering from a single arrival or a constructive addition of multiple arrivals whose separations in time are smaller than $1 / B$. This implication is very important in interpreting the scattering data when PC processing is involved.

\section{Partial wave target strength (PWTS)}

In Eq. (13), $F_{\text {bs }}$ is expressed as the total scattered wave from the target, without distinguishing between the various partial waves that make up that signal. To help understand the scattering mechanism of interest, in the case when the echoes (arrivals) can be resolved in the time domain, a partial wave target strength (PWTS) can be introduced to include only partial scattering waves from a subset of the scatterer:

$$
\mathrm{TS}_{p w}=20 \log _{10}\left|\sum_{j}^{N_{j}} F_{\mathrm{bs}}^{(j)}\right|,
$$

where $N_{j}$ is the number of partial waves of interest corresponding to the number of arrivals of the $\mathrm{CP}$ output. $F_{\mathrm{bs}}^{(j)}$ is the partial wave scattering amplitude or Fourier transform of a single ( $j$ th) arrival. By replacing $V_{\mathrm{bs}}^{(r)}$ and $V_{\text {cal }}^{(r)}$ in Eq. (14) with the Fourier transforms of their cross correlations with

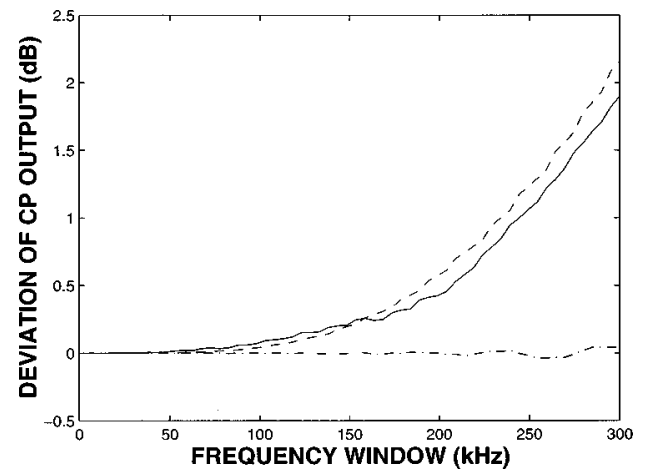

FIG. 4. Influence of the width of the frequency window on the target strength averaged over that window. The scattering amplitude, $F_{\text {bs }}(f)$ in Eqs. (24) and (25) is the Fourier transform of a triangle function with a 10 $\mu$ s spread. Three transmit signals are used in the computations: ideal (untapered) chirp (dash-dotted), Gaussian tapered chirp (dashed), and actually measured chirp (received signal in the calibration mode) used in the experiment (solid).

the transmit signal, $R_{\mathrm{bs}}^{(r)}$ and $R_{\text {cal }}^{(r)}$, respectively, the summation of all partial waves of interest is given by

$$
\sum_{j}^{N_{j}} F_{\mathrm{bs}}^{(j)}=\frac{V_{\mathrm{cal}}^{(t)}}{V_{\mathrm{bs}}^{(t)}} \frac{r_{\mathrm{bs}}^{2}}{r_{\mathrm{cal}}} \frac{\sum_{j}^{N_{j}} R_{\mathrm{bs}(j)}^{(r)}}{R_{\mathrm{cal}}^{(r)}},
$$

where $R_{\mathrm{bs}(j)}^{(r)}$ is the Fourier transform of the cross correlation of the $j$ th received scattering arrival with the calibration signal and can be chosen to include the partial waves of interest. $R_{\text {cal }}^{(r)}$ is the Fourier transform of the autocorrelation of the calibration signal. For a linear system, the ratio $V_{\text {cal }}^{(t)} / V_{\text {bs }}^{(t)}$ can be considered approximately as a constant over a usable frequency band.

In many cases, the highest SNR is more desirable than the precise partial wave target strength at a particular frequency. To obtain the highest SNR of a partial wave, we can use the peak value of the partial wave (arrival) obtainable from the CP output. As discussed in Sec. II C, this peak value corresponds to a weighted average of a single partial wave [Eq. (21)] in the frequency domain. The averaged scattering amplitude of such a partial wave ( $j$ th arrival) can be obtained directly from the $\mathrm{CP}$ output,

$$
\begin{aligned}
\left\langle\sigma_{\mathrm{bs}}^{(j)}\right\rangle & =\left\langle\left|F_{\mathrm{bs}}^{(j)}\right|^{2}\right\rangle, \\
& =\left\langle\left|\frac{V_{\mathrm{ca}}^{(t)}}{V_{\mathrm{bs}}^{(t)}} \frac{r_{\mathrm{bs}}^{2}}{r_{\mathrm{cal}}} \frac{R_{\mathrm{bs}(j)}^{(r)}}{R_{\mathrm{cal}}^{(r)}}\right|^{2}\right\rangle, \\
& =\left|\frac{V_{\mathrm{cal}}^{(t)}}{V_{\mathrm{bs}}^{(t)}} \frac{r_{\mathrm{bs}}^{2}}{r_{\mathrm{cal}}} \frac{y_{\mathrm{bs}(j)}^{(r)}\left(t_{j}\right)}{y_{\mathrm{cal}}^{(r)}}\right|^{2},
\end{aligned}
$$

where $\langle$.$\rangle stands for averaging over frequency, y_{\mathrm{bs}(j)}^{(r)}\left(t_{j}\right)$ is the cross correlation of the $j$ th received scattering arrival at $\tau=t_{j}$ time lag, and $y_{\mathrm{cal}_{\text {max }}}^{(r)}$ is the maximum peak value of the autocorrelation of the calibration signal. In Eqs. (29) and (30), index $j$ represents the partial wave scattering contribution from the $j$ th arrival in the time domain. Note that by assuming $V_{\mathrm{cal}}^{(t)} / V_{\mathrm{bs}}^{(t)}$ is a constant over the frequency band of interest, the average partial wave scattering cross section over the frequency band can be achieved by a simple ratio of CP outputs. The averaged PWTS from the $j$ th arrival can then be defined as 


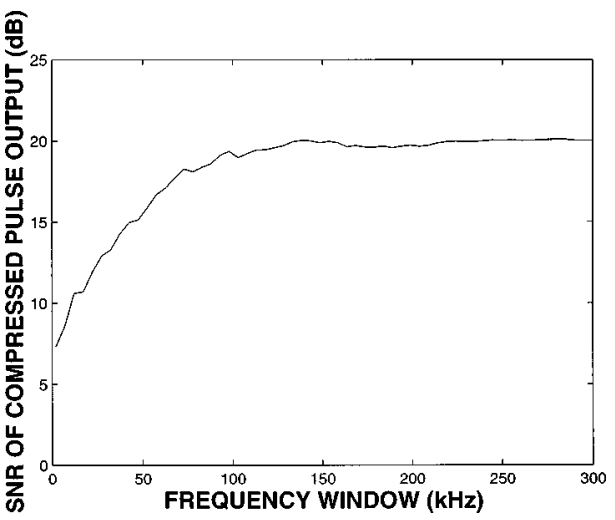

FIG. 5. Influence of the width of the frequency window on the output SNR of PC processing. The transmit signal is the measured signal shown in Fig. 2(e), while random noise is added to the original signal. The ratio of the signal amplitude (peak-to-peak) to the standard deviation of the noise is 5 and the resultant SNR is about $11 \mathrm{~dB}$. 100 realizations are used in the computation.

$$
\left\langle\mathrm{TS}_{p w}^{(j)}\right\rangle \equiv 10 \log _{10}\left\langle\left|F_{\mathrm{bs}}^{(j)}\right|^{2}\right\rangle
$$

From the discussion associated with Fig. 3, a possible error of up to several $\mathrm{dB}$ in estimating the "true" average target strength can be introduced if the spread of the "true" scattering impulse response is as large as a few micro seconds. However, for a scattering impulse response with a shorter length, the error is insignificant and may be neglected.

\section{DATA ANALYSIS AND DISCUSSIONS}

In this section, experimental scattering data from live individual zooplankton will be analyzed using three different approaches: temporal, spectral, and statistical analyses.

The data were collected during two ship cruises, September 27-October 5, 1993 and September 21-September 30, 1994 on or near Georges Bank (near Cape Cod, Massachusetts), and two series of laboratory tank experiments conducted at Woods Hole Oceanographic Institution (WHOI) in 1990 and at Naval Underwater Warfare Center (NUWC) in Newport, RI, from the end of 1991 to the beginning of 1992.

During the two cruises, a 1.5 -m-high by 2.4-m-diameter cylindrical tank was mounted on the deck of the ship. A transducer array was mounted on the bottom of the tank looking upward. The array comprised nine closely spaced transducer pairs for 1993 and 13 pairs for 1994, with frequencies ranging from $50 \mathrm{kHz}$ to $2 \mathrm{MHz}$ including four broadband transducer pairs whose center frequencies are 250 $\mathrm{kHz}, 500 \mathrm{kHz}, 1 \mathrm{MHz}$, and $2.25 \mathrm{MHz}$, respectively. Linear chirps were applied to all of the broadband transducers. Live animals were carefully tethered and put into the acoustic beam at a fixed range $(50 \mathrm{~cm}$ above the transducers) in the tank. One or more transducer pairs were used for obtaining the backscattering data from each animal. Detailed descriptions of the experimental setup and procedures can be found in Stanton et al. (1998a).

For the laboratory experiments, at NUWC, a one-cycle $500 \mathrm{kHz}$ pulse was applied to the transducer, while a chirp signal centered at $500 \mathrm{kHz}$ was used for the WHOI experiment [detailed descriptions of the experiments can be found in Chu et al. (1992) and Stanton et al. (1994a,b, 1998a)].

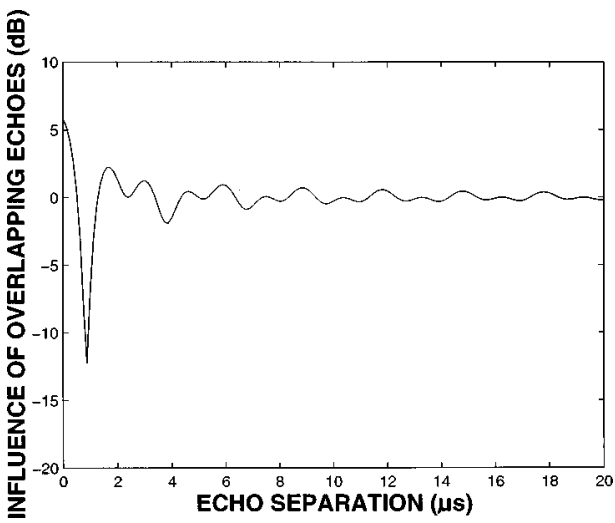

FIG. 6. Influence of the separation of two arrivals on the average target strength computed from Eq. (27). The transmit signal is an untapered chirp signal swept from $300 \mathrm{kHz}$ to $700 \mathrm{kHz}$ over $200 \mu$ s shown in Fig. 2(a).

The bandwidth of the transmitted chirp signal was $400 \mathrm{kHz}$ and the pulse length was $200 \mu$ s. Because of a much shorter pulse length used in the NUWC experiment, the PC processing gain is expected to be lower than that when using a chirp signal.

For all experiments, the calibrations were done in a bistatic mode with two transducers facing each other. In the scattering measurements, the configuration was still bistatic but the same transducers were mounted closely next to each other to approximate a true backscattering geometry.

In the following analysis, zooplankton from three anatomical groups are studied in the experiment, namely, shrimp-like animals (euphausiids and decapod shrimp) whose average length and diameter are about $30 \mathrm{~mm}$ and 4 $\mathrm{mm}$, respectively; gas-bearing animals (siphonophores) whose diameter of the gas inclusion and body length are about $1 \mathrm{~mm}$ and $30 \mathrm{~mm}$, respectively; and elastic shelled animals (gastropods) whose length and diameter are $2 \mathrm{~mm}$ and $1 \mathrm{~mm}$, respectively. The shapes of these animals can be found in Stanton et al. (1994b). These three animal groups correspondingly represent three different boundary conditions: fluid/fluid boundaries, a bubble embedded in a fluidlike body, and an elastic shell in a fluid medium. Since the transmit pulse length is only $200 \mu \mathrm{s}$, the Doppler shift due to animal movement can be ignored.

Before analyzing the $\mathrm{CP}$ output data in terms of the scattering physics of the targets, we need to examine the various sources of contamination in the experiment that could lead to errors or "false targets" in the output. The CP output that will be used in the following analysis includes two major arrivals: primary and secondary arrivals. For shrimplike animals such as euphausiids near broadside incidence, the primary arrivals (scattering from the front interface) and secondary arrivals (scattering from back interface) are often of comparable level and the contamination is insignificant. However, there is always a largest arrival in company with several smaller arrivals in the $\mathrm{CP}$ output for the siphonophores or gastropods. If we define the largest and second largest peaks as the primary and the secondary arrivals, respectively, it is found that the secondary arrivals are much weaker than the primary arrivals.

In general, the primary arrivals are reliable and easily identified while the secondary arrivals, except for euphaus- 
iids, are much weaker and exhibit larger variability in both arrival time and peak value. Therefore, they are more likely to be contaminated by echoes from the tether and microstructure, as well as system noise and artifacts of the PC processing itself (sidelobes). In addition to these possible sources of contamination, superglue was used to affix the tether to the gastropod which added another potential source of error [superglue was required when the animal was too small (1-2 $\mathrm{mm}$ long) to be tied to the tether]. Appendix D presents the results of the numerical simulations used to evaluate the error caused by the tether, microstructure, superglue, and the artifacts of the PC processing. It is found that even when a moderate noise component is added to the actual received signal, the major source of error is due to the artifacts of the PC processing. The artifacts can be greatly reduced if we only choose the pings in such a way that when the amplitude of the secondary arrival is greater than $10 \%$ of that of the primary arrival and when the secondary arrival occurs at points other than where the largest processing sidelobe occurs. In general, by using a PC technique, some low SNR backscattering data that were otherwise considered as unreliable in a spectral analysis can provide much useful information.

\section{A. Temporal characteristics-Resolving partial waves}

\section{Shrimplike animals (euphausiid and decapod shrimp)}

In a series of our previous studies, it was found that a shrimplike animal can be viewed as a weak scatterer and be modeled acoustically as the superposition of various rays:

$$
F_{\mathrm{bs}} \sim F_{\mathrm{FI}}+\sum_{j} b_{j} e^{i 2 k \epsilon_{j}},
$$

where $F_{\mathrm{bs}}$ is the total backscattering amplitude, $b_{j}$ is the amplitude of the $j$ th arrival and $\epsilon_{j}$ is the corresponding distance between the "acoustic center of mass" and the reference plane (zero phase plane). The first term $F_{\mathrm{FI}}$ corresponds to the scattering from the front interface of the animal, while the second term represents the total contributions from the other parts of the body (Stanton et al., 1993a, 1993b, 1998a). In the case of broadside or near broadside incidence, the second term quite often involves only one ray that penetrates the front interface into the body, bounces back from the back interface, passes through the front interface again and finally back to the receiver as shown in Fig. 7(a). The latter is shown to be reasonably approximated by $\mathrm{Re}^{i k s}$ (Stanton et al., 1993a, 1993b), where $R$ is the plane wave reflection coefficient from a plane interface, $k$ is the wave number, and $s$ is the round trip distance between the front and the back interfaces $[2 \overline{A B}$ in Fig. 7(a)]. Since the acoustic properties of the animal such as density and sound speed of the animal are very close to those of the surrounding fluid (water), the two rays have comparable strength. The phase difference of the two rays that arrive at the receiver is a function of frequency. In a plot of backscattering amplitude versus frequency, it produces an oscillatory pattern due to constructive (when the two rays are in phase) and destructive (when the two rays are out of phase) interferences. The nulls produced by destructive interactions could be as deep as $30 \mathrm{~dB}$ and sometimes have a regular spacing (Chu et al., 1992). Under other conditions when changes in shape and/or orientation give rise to multiple arrivals from different parts of the animal body, the pattern may be irregular (Stanton et al., 1998a). This variability in pattern has greatly limited our ability to accurately interpret broadband scattering data with a standard spectral analysis.

PC processing is an alternative way to approach this problem as it helps (temporally) resolve the various sources of scattering. Figure 8(a) shows a single ping backscattering signal from a 2-cm-long euphausiid, while Fig. 8(d) is its TS versus frequency plot. Partly due to the bandwidth limitation, no pattern can be clearly seen. In contrast, the $\mathrm{CP}$ output for the same ping is plotted in Fig. 8(g) showing two distinct arrivals with comparable strength. The time difference between the two arrivals corresponds to the round trip time required for the acoustic wave, after penetrating the animal body, traveling from the front interface to the back interface and returning. This result supports our previously proposed simple two-ray model that describes the (near broadside) backscattering from a weakly scattering elongated object such as a euphausiid (Stanton et al., 1993a, 1998b), i.e., the first arrival corresponds to the echo from the front interface while the second corresponds to the echo from the back interface. Note that the time difference between the two arrivals in Fig. 8(a) is much larger than the separation of the two arrivals shown in Fig. 8(g) (the former is about $65 \mu$ s and the latter is about $6.5 \mu$ s while the average cylindrical diameter of the animal is about $4 \mathrm{~mm}$ ). The null in the time series in Fig. 8(a) stems from a natural interference between two comparable chirp arrivals.

\section{Gas-bearing animals (Siphonophore)}

A siphonophore has a gas inclusion embedded in an elongated weakly scattering body. The acoustic scattering model for a siphonophore can be written approximately as

$$
F_{\mathrm{bs}} \sim F_{\mathrm{GAS}}+F_{\mathrm{TISSUE}},
$$

where $F_{\mathrm{GAS}}$ and $F_{\mathrm{TISSUE}}$ represent scattering from the gas inclusion and body tissue, respectively. The second term on the right-hand side of Eq. (33) is the superposition of all rays scattered from the body tissue. A schematic scattering diagram for a siphonophore is illustrated in Fig. 7(b). A typical time series, its spectrum, and the corresponding CP output are shown in Fig. 8(b), (e), and (h), respectively. The largest peak of the CP output from this animal is due to the backscattering from the gas inclusion. The second largest peak which arrives before the main peak (pre-arrival) is interpreted as the scattering from the body tissue. This interpretation was based on the observation using a video camera that the main tissue portion of the animal was closer to the transducer than the bubble inclusion for these data. A time difference of $16.5 \mu$ s obtainable from Fig. 8(h) corresponds to a spatial distance of about $12.4 \mathrm{~mm}$. This distance can be interpreted as the distance between the gas bubble and the "local acoustic center of mass" of the body [d in Fig. 7(b)]. This "local acoustic center of mass" could be a large glint or the superposition of several glints arriving at approximately the same time. The spatial distance of $12.4 \mathrm{~mm}$ is a 


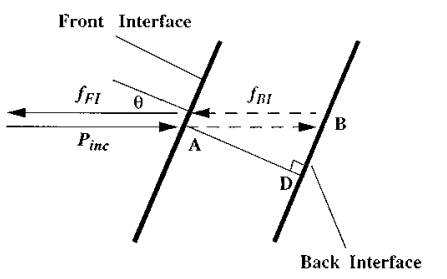

(a)

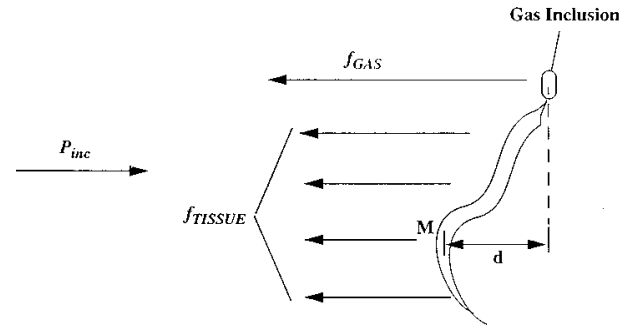

(b)

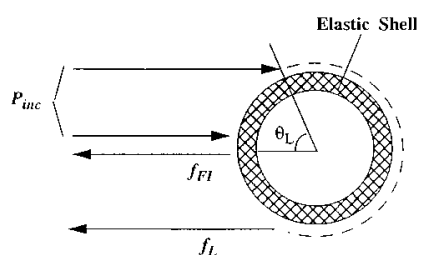

FIG. 7. Schematic diagram of scattering mechanisms for animals from three different zooplankton groups. (a) Euphausiid; (b) siphonophore; and (c) gastropod.

reasonable number for the curled animal whose body length is $26 \mathrm{~mm}$ when fully extended.

\section{Elastic shelled animals (Gastropod)}

A gastropod is a marine snail and has a hard elastic shell. For such a hard-shelled object, the incident wave hardly penetrates the shell or is greatly attenuated. However, other types of acoustic waves can be generated with a shell. A typical time series, its spectrum and its $\mathrm{CP}$ output are plotted in Fig. 8(c), (f), and (i), respectively. The primary arrival in the $\mathrm{CP}$ output plot (the largest peak) corresponds to the specular component of the backscattering from the front interface much like the cases for the euphausiid and siphonophore. There is also a secondary arrival, the event following the primary arrival (circled peak), corresponding to another kind of acoustic wave. For a time difference of $15 \mu$ s (round trip) measured in Fig. 8(i), assuming a sound speed of 1500 $\mathrm{m} / \mathrm{s}$, the corresponding spatial distance is $11.3 \mathrm{~mm}$. This spatial distance is much larger than the outer physical dimensions (the animal is about $2 \mathrm{~mm}$ long and $1 \mathrm{~mm}$ in diameter with a shell thickness about $5 \mu \mathrm{m}$ ) of the gastropod from which the data were collected.

One reasonable explanation is that the hard elastic shell is capable of supporting surface elastic waves that travel at a speed lower than that of the sound speed in the surrounding fluid (Stanton et al., 1998b). This subsonic wave is known as the zeroth order antisymmetric Lamb wave (Zhang et al., 1992). For a subsonic surface wave, the "landing/ launching" angle $\theta_{L}$ is $\pi / 2$; i.e., the acoustic wave that impinges on the shell tangentially will generate a subsonic
Lamb wave that circumnavigates the elastic shell and sheds back to the receiver as shown in Fig. 7(c).

As a result, the acoustic backscattering model for this hard shell object can be expressed as

$$
F_{\mathrm{bs}} \sim F_{\mathrm{FI}}+F_{\mathrm{Lamb}}
$$

where $F_{\mathrm{FI}}$ and $F_{\mathrm{Lamb}}$ represent scattering from the front interface of the animal and from the subsonic Lamb wave, respectively. Note that besides the two major peaks, there are some smaller arrivals in Fig. 8(i). These peaks are possibly due to scattering by a combination of other features of the animal and microstructure as well as from sidelobe artifacts of the PC processing discussed in Appendix D.

\section{B. Spectral characteristics-Improving the SNR}

As discussed above, if there is more than one arrival from the target, the signal $f_{\text {bs }}(t)$ can be decomposed into a sum of a number of arrivals described in Eq. (19). If, by using PC processing, the echoes from different parts of an animal as well as from other scatterers such as microstructure can be resolved in the time domain, the time series can be gated in such a way that only the arrivals of interest are included. Such a gating rejects the resolvable unwanted arrivals which include echoes from microstructure and partial waves that may not be of interest. If we treat the unwanted arrivals together with the background white noise as the total effective noise, the resultant SNR could be improved from $E_{w} /\left(N_{\text {white }}+E_{u w}\right)$ to $E_{w} / N_{\text {white }}$, where $E_{w}$ and $E_{u w}$ are energies of wanted and unwanted arrivals, respectively, and $N_{\text {white }}$ is the power density of the white noise.

The time series for three different zooplankton groups and their corresponding spectra are shown in Fig. 9, where the truncated time series and their corresponding spectra are plotted with thicker solid lines. For each animal, only two arrivals or partial waves are chosen, i.e., $N_{j}=2$ in Eq. (28). The spectra of the truncated time series can be considered as PWTS defined in Eq. (28). These spectra (thicker lines) are optimized outputs in that only the two major arrivals that dominate the scattering are included and most of the unwanted echoes stemming mainly from microstructure reverberation as well as some in-band noise are windowed out. As a result of the truncation, the hashy structure of the original spectra (thinner lines) has been removed, which indicates an improved SNR of the desired signal. The resultant spectra clearly illustrate the interferences between two rays, especially for the siphonophore whose original spectrum was too noisy to see the regular interference pattern due to the two rays.

In Fig. 9, since only one rectangular time window is used for each time series, we refer to this process as a "truncation." However, in general, it is more appropriate to use the terminology "windowing" or "gating" to describe the process since multiple time windows can be used to include any number of wanted arrivals as long as the arrivals can be resolved. The number of time windows used in the process and the type of windowing depends on the purpose of the analysis.

Since spectral analysis automatically eliminates the outof-band noise in the frequency domain while windowing of 
EUPHAUSIID
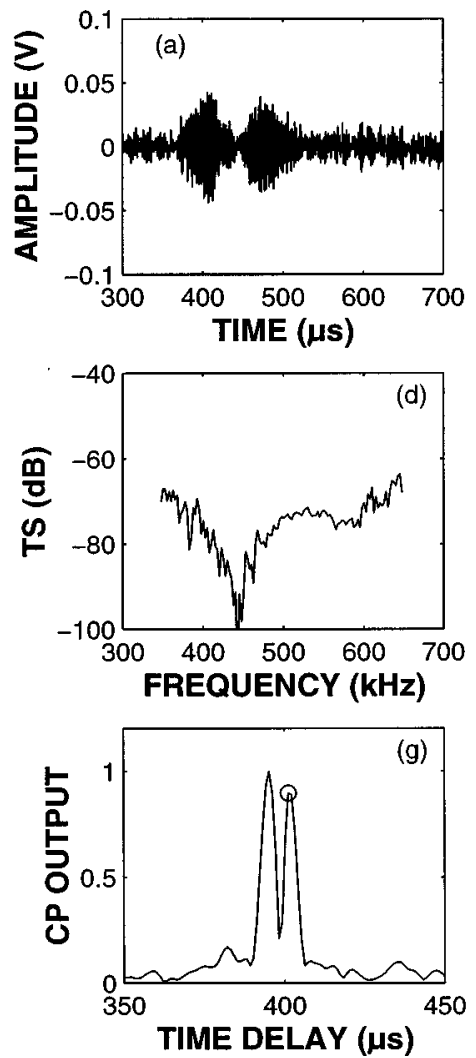

SIPHONOPHORE
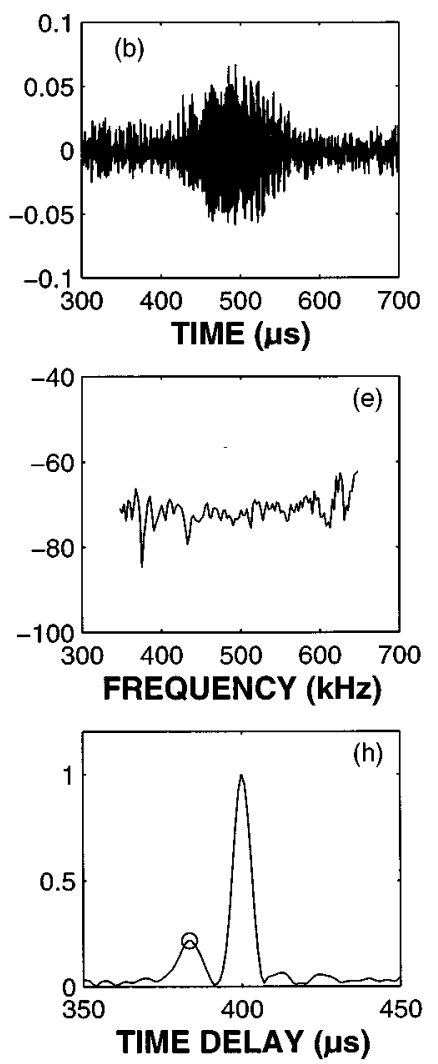

GASTROPOD
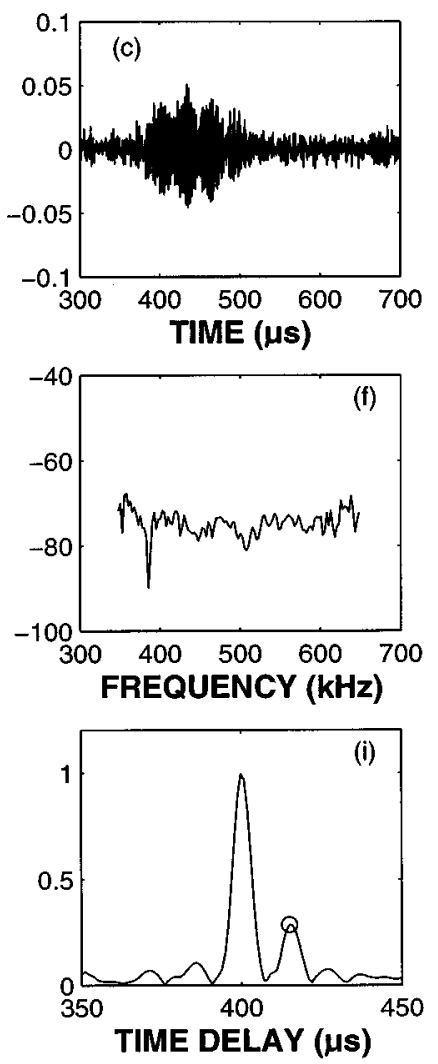

FIG. 8. Single ping analysis from three different zooplankton groups. (a), (d), and (g) for euphausiid; (b), (e) and (h) for siphonophore, and (c), (f) and (i) for gastropod. First row is the time series, second row is the target strength versus frequency, and the third row is the envelope of the CP output where the circled peaks are the secondary arrivals. The transmit signal is a chirp signal shown in Fig. 2(e). Note that the separation between the two major peaks in (g) $(6.0 \mu)$ is much smaller than that observed in the corresponding time series in (a) (65 $\mu \mathrm{s})$.

the $\mathrm{CP}$ output can filter out some unwanted in-band signals in the time domain, the resultant PWTS in this process is expected to have a better output SNR as shown in Fig. 9.

\section{Statistical characteristics}

Since the scattering by live animals is a stochastic process, a statistical analysis of the time series of the CP output is required. This analysis provides at least two types of information: distributions of time difference between two major arrivals and their amplitude ratio (Tables I and II). To eliminate data with unacceptably high contamination due to noise and inherent sidelobe artifacts of the PC processing, we reject the pings whose SNR is below a certain level and whose amplitude ratio of the primary arrival (the largest peak) to the secondary arrival (the second largest peak) is larger than a preset threshold. This threshold is chosen to be the ratio of the main lobe to the largest sidelobe when the PC processing is performed on the received chirp signal in the calibration mode with no target present. As shown in Fig. 2(f), this ratio is about 10 in a noise-free situation and is purely due to the (processing sidelobe) artifacts of the PC operation (Appendix D).

\section{Animal characterization}

The histograms of the time difference and the amplitude ratio of primary-to-secondary arrivals for animals from three different zooplankton groups are shown in Fig. 10.
For a euphausiid, the histogram of the time difference between the first and second arrivals is narrowly distributed [Fig. 10(a)]. In contrast, the histograms for a 26-mm-long siphonophore [Fig. 10(c)] and a 2-mm-long gastropod [Fig. $10(\mathrm{e})]$ are more spread out and peaked at values greater than $10 \mu \mathrm{s}$.

In terms of the amplitude histogram, the euphausiid has the narrowest distribution (note that the scale for the euphausiid is different from those for the siphonophore and gastropod), and the siphonophore has the widest amplitude distribution. Table I summarizes the statistics of the time differences and amplitude ratios for the three different animals shown in Fig. 10.

\section{Sizing animals}

Certain dimensions of the animals can be estimated by examining the temporal separation between $\mathrm{CP}$ arrivals and relating those times to the dimensions using a scattering model. The accuracy of the estimates are limited, in part, by the temporal resolution of the signal. For example, for a chirp signal with a bandwidth of $250 \mathrm{kHz}$ (a composite bandwidth due to the transmitter/receiver combination), the duration of the received signal can be compressed to about $4 \mu \mathrm{s}$, which corresponds to a spatial resolution of $3 \mathrm{~mm}$ in water for backscattering.

The histogram of the time difference and the amplitude ratio of primary-to-secondary arrivals from 200 pings for a 


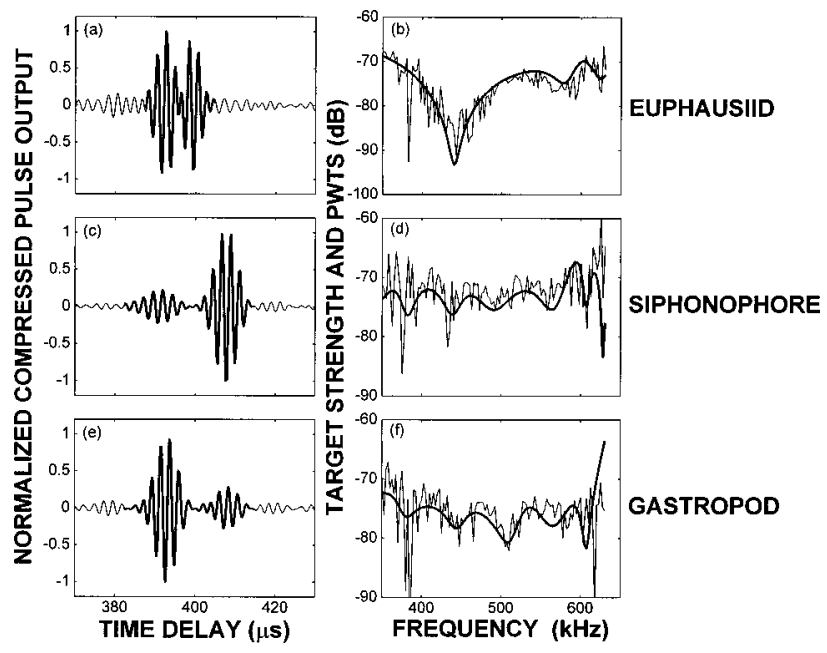

FIG. 9. Partial wave target strength (PWTS) for animals from three different zooplankton groups. (a) and (b) for euphausiid; (c) and (d) for siphonophore; and (e) and (f) for gastropod. The plots on the left column are normalized CP outputs (no demodulation). The thin lines are the original CP outputs while the thick lines are the filtered CP outputs using an optimum rectangular time window. The plots on the right column are TS (thin lines) and PWTS (thick lines). The TS is computed using Eq. (C2) while the PWTS is calculated using Eq. (28) and involves the filtered CP outputs. The transmit signal for all three animals is a chirp signal shown in Fig. 2(e). The partial wave analysis involves a window that includes the two main echoes and filters out echoes or noise outside the window.

25-mm-long euphausiid (meganyctiphanes) are shown in Fig. 10(a) and (b) (first row). The minimum value of the time difference in the histogram is $4.74 \mu \mathrm{s}$. Assuming that the minimum time difference corresponds to the case when the animal is oriented at broadside incidence, we use this value to estimate the average cylindrical diameter of this weakly scattering animal to be $3.6 \mathrm{~mm}$ (which is the same as the measured diameter of $3.6 \mathrm{~mm}$ ). This agreement of the inferred animal size with that actually measured indicates that it is possible to size animals by using a PC technique. In the amplitude ratio histogram, the maximum value is around 1.1, which is a reasonable value for a weakly scattering target.

Table II lists the comparisons between the measured diameters of euphausiids and decapod shrimp and those computed from the time difference corresponding to the peak value of the histogram of the CP outputs similar to Fig. 10(a) and (b). Due to a combination of system noise and the limitation of the temporal resolution, the peak (mode) histogram values were used for Table II rather than the minimum values. The data were collected during the two cruises on Georges Bank and the two laboratory tank experiments as described at the beginning of this section. From Table II, we see that except for sample \#4 the size of all animals are overestimated. This is due, in part, to the fact that the peak (mode) histogram values were used instead of minimum values. Another contribution to an overestimation is from the fact that the least time difference corresponds to the case in which the incidence is truly broadside $[\theta=0$ in Fig. 7(a) $]$. For all angles of incidence away from broadside, the dimension of the cross section in the incident plane is increased by a factor of $1 / \cos \theta$ resulting in an increased travel time.

For the NUWC data (sample \#4), since only a one-cycle pulse $(2 \mu \mathrm{s})$ was used, the output SNR was lower (since the
TABLE I. Summary of statistics for three different animal groups. $\Delta_{t}:$ time difference between two arrivals in CP output (minimum time for euphausiid), $\sigma_{t}$ : standard deviation of $\Delta_{t}, \rho_{a}$ : amplitude ratio of two arrivals, and $\sigma_{a}:$ standard deviation of $\rho_{a}$.

\begin{tabular}{lccc}
\hline \hline & Euphausiid & Gastropod & Siphonophore \\
\hline$\Delta_{t}(\mu \mathrm{s})$ & 4.74 & 17.37 & $(-) 14.21$ \\
$\sigma_{t}(\mu \mathrm{s})$ & 0.72 & 3.77 & 6.80 \\
$\rho_{a}$ & 1.03 & 5.65 & 6.67 \\
$\sigma_{a}$ & 0.47 & 1.29 & 1.98 \\
\hline \hline
\end{tabular}

SNR is proportional to the product of the bandwidth and the pulse length as discussed in Sec. I) and possible noise contamination in the sizing result is expected. The final overall results still agree reasonably well with the measured data, with relative errors less than $28 \%$.

The histograms of the time difference and the amplitude ratio from 200 pings for a siphonophore are shown in Fig. 10(c) and (d) (second row). In plotting the histograms, we have used the "pre-arrivals" as the chosen secondary echoes representing the scattering from the body tissue since the video camera recording reveals that the main portion of the body tissue of the animal was closer to the transducer than the gas inclusion. The time difference at the peak of the histogram corresponds to a separation of $10.7 \mathrm{~mm}$ which should be compared with the 26-mm body length of the animal. The animal was partially curled during the experiment and the $10.7-\mathrm{mm}$ value is consistent with scattering off an intermediate part of the body. Further studies show that the time differences between the main peak and "post-arrival" are more randomly distributed and the amplitudes of the latter are lower than those of the pre-arrivals which suggests that post-arrivals are likely resulting from randomly distributed micro-structure.

Figure 10(e) and (f) shows the histograms of the time difference and the amplitude ratio for a gastropod. As previously discussed, the secondary arrival is always a postarrival since it corresponds to a subsonic Lamb wave that circumnavigates the elastic shell and then sheds back to the receiver and always arrives later than the primary arrival. Further work is required toward understanding the variability of the speed of the subsonic wave with respect to acoustic

TABLE II. Comparison of animal size between measured and that inferred ("computed") from CP output. The inferred animal sizes are the values corresponding to the peaks (mode values) of the histograms based on measured time differences between primary and secondary arrivals from $\mathrm{CP}$ outputs.

\begin{tabular}{ccccc}
\hline \hline Sample \# & Species & $\begin{array}{c}\text { Measured } L \\
(\mathrm{~mm})\end{array}$ & $\begin{array}{c}\text { Measured }\langle D\rangle \\
(\mathrm{mm})\end{array}$ & $\begin{array}{c}\text { Inferred }\langle D\rangle \\
(\mathrm{mm})\end{array}$ \\
\hline 1 & Euphausiid $^{\mathrm{a}}$ & 30.0 & 3.6 & 4.7 \\
2 & Euphausiid $^{\mathrm{a}}$ & 30.3 & 3.7 & 4.8 \\
3 & Euphausiid $^{\mathrm{b}}$ & 34.0 & 3.5 & 4.8 \\
4 & Decapod Shrimp $^{\mathrm{c}}$ & 25.0 & 4.5 & 3.9 \\
5 & Decapod Shrimp $^{\mathrm{d}}$ & 17.8 & 3.0 & 3.8 \\
6 & Decapod Shrimp $^{\mathrm{d}}$ & 26.1 & 4.4 & 4.4 \\
\hline \hline
\end{tabular}

${ }^{a} 1994$ cruise data (shipboard).

${ }^{\mathrm{b}} 1993$ cruise data (shipboard).

'October 1992-February 1993 NUWC laboratory data (on land).

d1990 WHOI laboratory data (on land). 


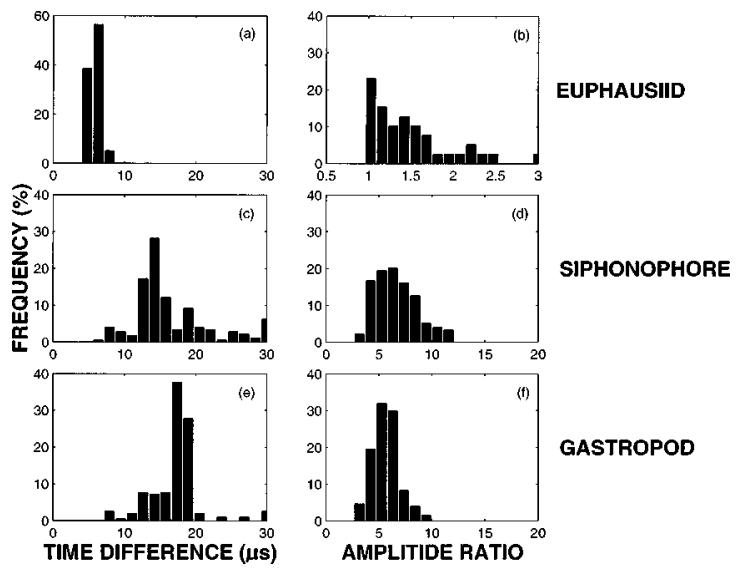

FIG. 10. Statistics of the CP outputs for animals from three different zooplankton groups. (a) and (b) for euphausiid; (c) and (d) for siphonophore; (e) and (f) for gastropod. The plots in the left column are histograms of time difference between the primary and secondary arrivals while the plots on the right are histograms of the peak amplitude ratios of the primary to the secondary arrivals. The transmit signal for all three animals is a chirp signal shown in Fig. 2(e)

frequency, shell thickness, size, orientation, and material properties before we can assess our ability to estimate size from the time difference information.

\section{Relation between averaged TS and PWTS}

Another interesting result is the comparison of the backscattering by the siphonophore with and without its gas inclusion. About 5-dB difference in average target strength was observed in Stanton et al. (1998a). The statistics of CP outputs for the siphonophore with and without gas is shown in Fig. 11, where the left three plots (first column) are for the siphonophore with gas as indicated in the figure while the other three are for the siphonophore without gas. Since the backscattering for the siphonophore without gas is weaker, to improve the SNR and obtain a more reliable estimate, we have used Eq. (31) to compute the PWTS of the largest arrival averaged over a frequency band. The largest arrivals for the two sets of data analyzed: one corresponds to the echoes from the gas when the whole (with gas) siphonophore is involved, and the other corresponds to the tissue when the gas-less animal is involved. Comparison of Fig. 11 and Fig. 5 in Stanton et al. (1998a) shows that the average values of TS for the siphonophore with and without its gas inclusion are about $1.7 \mathrm{~dB}$ and $6.7 \mathrm{~dB}$ higher than the averaged PWTS of the largest arrivals in the corresponding cases shown in Fig. 11(c) and (d). These differences can be explained as follows: for the siphonophore with its gas inclusion, since only the primary arrival (scattering from gas inclusion) is used to compute the averaged PWTS, the contribution from the body tissue is excluded. From Stanton et al. (1998b), it is found that the body tissue contributes about $1 / 3$ of the total scattered energy. Thus excluding the body tissue scattering will result in a $1.8-\mathrm{dB}$ drop $\left[10 \log _{10}(2 / 3)\right]$ in estimated PWTS, which is close to the measured value of $1.7 \mathrm{~dB}$.

For the case without the gas inclusion, Stanton et al. (1998b) used six rays with equal scattering strength but with randomly and uniformly distributed phases to simulate the statistical nature of the backscattering from the body tissue (no gas) of a siphonophore. If we choose one of these six rays to be the primary arrival (it is considered as the secondary arrival in the case where the gas inclusion is not removed), the exclusion of the other five rays would make the averaged PWTS about $7.8 \mathrm{~dB}\left(10 \log _{10} 6\right)$ lower than the case when all rays are included [Fig. 5 in Stanton et al. (1998a)]. This predicted 7.8-dB difference based on the sixray model with equal strength cannot explain a 6.7-dB reduction in the averaged PWTS as shown in Fig. 11(d). The six-ray model used in Stanton et al. (1998b) was satisfactory to describe the scattering from the body tissue of the animal since it agreed with the measured echo (Rayleigh-like) PDF reasonably well.

Further simulations show that the degree of agreement between data and the model is not inherently very sensitive to the number of rays involved in the statistical model as long as the number of rays exceeds five. However, since Fig. 11 is generated from the dominant ray only, the difference between the averaged TS and the averaged PWTS of the dominant arrival (scattering from the "local acoustic center of mass") is very sensitive to the number of rays that make up the total echo. A 6.7-dB difference mentioned above implies an $80 \%$ energy loss, which in turn suggests that a model with five rays of equal strength is more plausible in describing the scattering from the body tissue of this particular gas-bearing siphonophore under these experimental conditions. The echo amplitude PDF from five rays with equal strength is also Rayleigh-like (but less so than with six rays). The inclusion of only one out of five rays results in a $10 \log _{10}(5) \simeq 7.0$ - $\mathrm{dB}$ reduction in PWTS, which is a reasonable value as compared with the actually measured value of $6.7 \mathrm{~dB}$. This difference in number of rays to model the scattering by the tissue does not affect the results in Stanton et al. (1998b) given the granularity of the measured echo PDFs.

From Fig. 10(c) and (d), it can be seen that the histograms of the scattering amplitude are not Rician PDFs [which well described the total echoes at a particular frequency in Stanton et al. (1998b)]. This is because the data used to generate the histograms are obtained from the $\mathrm{CP}$ outputs (peak value) which is the integration over a frequency band, whereas a Rician PDF describes the echo statistics for CW-like signals.

\section{SUMMARY}

Pulse compression processing is a powerful tool for applications of acoustic scattering by live individual zooplankton as it improves the output SNR and temporal resolution of the echoes. Characteristics of the $\mathrm{CP}$ outputs of broadband scattering signals are studied in terms of the features of the scattered signals from acoustic targets. Broadband acoustic backscattering data from various experiments involving three animal groups are analyzed using the PC technique. The indepth studies of temporal, spectral, and statistical characteristics of the $\mathrm{CP}$ outputs are presented in this paper. The following conclusions are made based on these studies:

(1) The results presented in this paper support our previously proposed ray models for the three different zooplankton groups based on the dominant scattering mecha- 

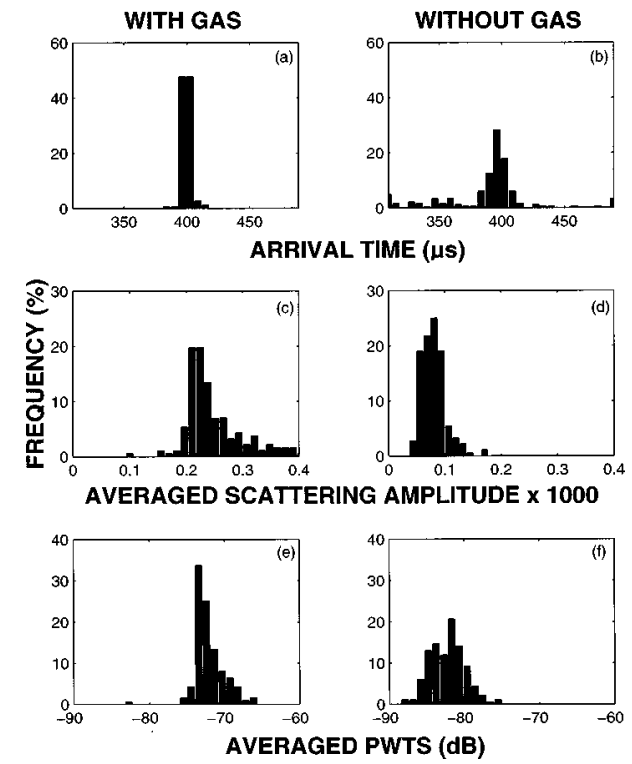

FIG. 11. Statistics of CP outputs from a siphonophore with and without gas inclusion. The averaged scattering amplitude [Eq. (30)] and its corresponding PWTS [Eq. (31)] are computed using the peak value of the primary arrival. The transmit signal is a chirp signal shown in Fig. 2(e).

nisms which depend on the different boundary conditions: (a) Shrimplike animals: one ray from the front interface and the others from other parts of the body. For near broadside incidence, a two-ray model adequately describes the dominant scattering features much of the time, where one ray is from the front interface and the other is from the back interface; (b) gas-bearing animals: one ray from the gas inclusion and the others from body tissue, the latter can sometimes be approximately represented by an equivalent ray scattered from the "local acoustic center of mass" of the body tissue; and (c) elastic shelled animals: one ray from the front interface and the other from the circumferential subsonic Lamb wave.

(2) Using an optimum window function based on the $\mathrm{CP}$ output, a partial wave contribution can be obtained to only include the dominant scattering features of interest and to eliminate certain unwanted signals. The TS based on such a windowed partial wave(s) can be characterized by the PWTS. As a result, the temporally gated CP output has been shown to dramatically improve the SNR of the corresponding frequency spectrum. A prerequisite condition for the windowing is that the echoes observed in the $\mathrm{CP}$ output must be resolved.

(3) The statistical studies on CP outputs demonstrate the ability of using the PC technique to size an individual animal and to differentiate zooplankton from different groups. The distinct characteristics of zooplankton from different groups (Table I) suggest the possible applications of the PC technique to zooplankton classification.

(4) The comparative study of the averaged PWTS versus the average TS of gas-bearing animals (siphonophore) allows a more quantitative estimate of the energy partition of the partial waves scattered from a single target and helps improve our understanding of the scattering mechanism.

The results presented in this paper have broader appli- cations in that this technique can easily be extended to other areas such as fisheries acoustics.

\section{ACKNOWLEDGMENTS}

This work was supported by the National Science Foundation under Grant No. OCE-9201264 and the U.S. Office of Naval Research under Grant Nos. N00014-89-J-1729, N00014-94-1-0452, and N00014-95-1-0287. This is Woods Hole Oceanographic Institution contribution No. 9413.

\section{APPENDIX A}

For a chirp signal with a Gaussian envelope defined in Eq. (8), where the function $u(t)$ is given by Eq. (6), we can express a cosine by two exponential functions

$$
\cos z=\frac{e^{i z}+e^{-i z}}{2}
$$

The autocorrelation function of $u(t)$ is

$$
\begin{aligned}
& R(\tau)=\frac{1}{T} \int_{-\infty}^{\infty} u^{*}(t-\tau) u(t) w(t) d t, \\
& =\frac{1}{4 T} \int_{-\infty}^{\infty}\left(e^{i \phi_{0}-A_{0}}+e^{-i \phi_{0}-A_{0}}\right)
\end{aligned}
$$

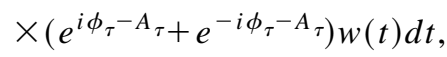

$$
\begin{aligned}
& =R_{1}(\tau)+R_{2}(\tau)+R_{3}(\tau)+R_{4}(\tau),
\end{aligned}
$$

where $w(t)$ is a window function that depends on the pulse length $T$ and the time delay variable $\tau$. The above terms are defined as follows:

$$
\begin{aligned}
& \phi_{0}=\omega_{0} t+\alpha t^{2}, \quad \phi_{\tau}=\omega_{0}(t-\tau)+\alpha(t-\tau)^{2}, \\
& A_{0}=\beta(t-T / 2)^{2}, \quad A_{\tau}=\beta(t-\tau-T / 2)^{2},
\end{aligned}
$$

and

$$
\begin{aligned}
& R_{1}(\tau)=\frac{1}{4 T} \int_{-\infty}^{\infty} e^{i\left(\phi_{0}+\phi_{\tau}\right)-A_{0}-A_{\tau}} \mathcal{W}(t) d t, \\
& R_{2}(\tau)=\frac{1}{4 T} \int_{-\infty}^{\infty} e^{i\left(\phi_{0}-\phi_{\tau}\right)-A_{0}-A_{\tau}} \mathcal{W}(t) d t, \\
& R_{3}(\tau)=\frac{1}{4 T} \int_{-\infty}^{\infty} e^{-i\left(\phi_{0}+\phi_{\tau}\right)-A_{0}-A_{\tau}}(t) d t, \\
& R_{4}(\tau)=\frac{1}{4 T} \int_{-\infty}^{\infty} e^{-i\left(\phi_{0}-\phi_{\tau}\right)-A_{0}-A_{\tau}}(t) d t .
\end{aligned}
$$

Since $R_{3}(\tau)=R_{1}^{*}(\tau)$ and $R_{4}(\tau)=R_{2}^{*}(\tau)$, Eq. (A2) is then

$$
R(\tau)=2 \operatorname{Re}\left\{R_{1}(\tau)+R_{2}(\tau)\right\} .
$$

For simplicity, we use a rectangular window function $w(t)$ of width $T$. Substituting Eq. (A3) into Eq. (A4) and rearranging the exponential of the integrand, we have 


$$
\begin{aligned}
R_{j}(\tau) & =\frac{1}{4 T} \int_{-\infty}^{\infty} e^{i\left(A_{j} t^{2}+B_{j} t+D_{j}\right)} \omega(t) d t, \\
& =\frac{1}{4 T} \int_{0}^{T-\tau} e^{i\left(A_{j} t^{2}+B_{j} t+D_{j}\right)} d t
\end{aligned}
$$

where $j=1,2$ and

$$
\begin{aligned}
& A_{1}=2(\alpha+i \beta), \quad A_{2}=2 i \beta, \\
& B_{1}=2 \omega_{0}-2 \alpha \tau-2 i \beta(T+\tau), \\
& B_{2}=2 \alpha \tau-2 i \beta(T+\tau), \\
& D_{1}=\alpha \tau^{2}-\omega_{0} \tau+i \beta\left(\frac{T^{2}}{2}+\tau^{2}+T \tau\right), \\
& D_{2}=-\alpha \tau^{2}+\omega_{0} \tau+i \beta\left(\frac{T^{2}}{2}+\tau^{2}+T \tau\right) .
\end{aligned}
$$

Equation (A6) can be rearranged into the form of a Fresnel integral with a complex argument,

$$
\begin{aligned}
F(z) & =\int_{z_{1}}^{z_{2}} e^{i(\pi / 2) w^{2}} d w \\
& =C\left(z_{2}\right)+i S\left(z_{2}\right)-C\left(z_{1}\right)-i S\left(z_{1}\right),
\end{aligned}
$$

where $C(z)$ and $S(z)$ are sine and cosine Fresnel integrals (Abramowitz and Stegun, 1965). Equation (A5) becomes

$$
\begin{aligned}
R(\tau)= & \frac{1}{2 T} \sum_{j=1}^{2} \operatorname{Re}\left\{\sqrt { \frac { \pi } { 2 A _ { j } } } e ^ { i ( 4 A _ { j } D _ { j } - B _ { j } ^ { 2 } ) / 4 A _ { j } } \left[C\left(H_{U j}\right)\right.\right. \\
& \left.\left.-C\left(H_{L j}\right)+i S\left(H_{H j}\right)-i S\left(H_{L j}\right)\right]\right\},
\end{aligned}
$$

where

$$
\begin{aligned}
& H_{U j}=\left(T-\tau+\frac{B_{j}}{2 A_{j}}\right) \sqrt{\frac{2 A_{j}}{\pi}}, \\
& H_{L j}=\frac{B_{j}}{2 A_{j}} \sqrt{\frac{2 A_{j}}{\pi}} .
\end{aligned}
$$

Since $\left|R_{j}(\tau)\right| \propto \sqrt{\pi / 2\left|A_{j}\right|}$, for $\alpha \gg \beta, R_{2}(\tau) \gg R_{1}(\tau)$, Eq. (A9) can be expressed approximately as

$$
\begin{aligned}
R(\tau) & \approx \frac{1}{2 T} \operatorname{Re}\left\{\sqrt{\frac{\pi}{2 A_{2}}} e^{i\left(4 A_{2} D_{2}-B_{2}^{2}\right) / 4 A_{2} F_{s c}}\right\}, \\
& =\frac{1}{4 T} \sqrt{\frac{\pi}{\beta}} e^{-\left[\left(\beta^{2}+\alpha^{2}\right) / 2 \beta\right] \tau^{2}} \operatorname{Re}\left\{e^{i(\omega+\alpha T) \tau-i \pi / 4} F_{s c}\right\}
\end{aligned}
$$

where

$$
F_{s c}=C\left(H_{U 2}\right)-C\left(H_{L 2}\right)+i S\left(H_{U 2}\right)-i S\left(H_{L 2}\right)
$$

and

$$
\begin{aligned}
& H_{U 2}=\sqrt{\frac{2 i \beta}{\pi}}\left(\frac{T-3 \tau}{2}-i \frac{\alpha}{2 \beta} \tau\right), \\
& H_{L 2}=\sqrt{\frac{2 i \beta}{\pi}}\left(-\frac{T+\tau}{2}-i \frac{\alpha}{2 \beta} \tau\right) .
\end{aligned}
$$

It can be shown that $F_{s c} \approx \sqrt{2} e^{i \pi / 4}$, thus we can obtain an approximate expression for $R(\tau)$

$$
R(\tau) \approx \frac{1}{4 T} \sqrt{\frac{2 \pi}{\beta}} e^{-\left[\left(\beta^{2}+\alpha^{2}\right) / 2 \beta\right] \tau^{2}} \cos \left[\left(\omega_{0}+\alpha T\right) \tau\right] .
$$

It can be seen that $R(\tau)$ is a Gaussian modulated sine wave with a carrier angular frequency $\omega_{0}+\alpha T$, which is the same as that for an ideal chirp case given by Eq. (7) but with a Gaussian envelope,

$$
R(\tau)_{\mathrm{env}}=\frac{1}{4 T} \sqrt{\frac{2 \pi}{\beta}} e^{-\left[\left(\beta^{2}+\alpha^{2}\right) / 2 \beta\right] \tau^{2}},
$$

which is the same as that given by Cook (1967).

This result shows that the envelope of the CP output of a Gaussian envelope chirp is also Gaussian when the leading and trailing edges of the chirp signal are smoothly varying. Equation (A14) can also be obtained by applying the method of steepest descent to Eq. (A6).

\section{APPENDIX B}

For real functions $f(t)$ and $g(t)$, the correlation function $r_{f g}(\tau)$ is defined as

$$
\begin{aligned}
r_{f g}(\tau) & =f(t) \otimes g(t), \\
& =\int_{-\infty}^{\infty} f(t-\tau) g(t) d t .
\end{aligned}
$$

By denoting $F(f)$ and $G(f)$ as the Fourier transforms of $f(t)$ and $g(t)$, it is well known that

$$
\begin{aligned}
& f(t) * g(t) \Leftrightarrow F(f) G(f), \\
& f(t) \otimes g(t) \Leftrightarrow F(-f) G(f),
\end{aligned}
$$

where the operation symbols " $*$ ', and " $\otimes$ " stand for convolution and correlation, respectively. The symbol " $\Leftrightarrow$ ', represents equivalency between the different domains (time and frequency). Since $f(t)$ is a real function, we have

$$
F(-f)=F^{*}(f),
$$

where $F^{*}$ is the complex conjugate of $F$. Using Eqs. (B1)(B3), we can obtain the following relation:

$$
\begin{aligned}
f(t) *(g(t) \otimes h(t)) & \Leftrightarrow F(f) G^{*}(f) H(f) \\
& =\left(F^{*}(f) G(f)\right)^{*} H(f) \\
& \Leftrightarrow(f(t) \otimes g(t)) \otimes h(t) .
\end{aligned}
$$

Thus

$$
f(t) *(g(t) \otimes h(t))=(f(t) \otimes g(t)) \otimes h(t) .
$$

Similarly, we can derive

$$
(f(t) * g(t)) \otimes h(t)=f(t) \otimes(g(t) \otimes h(t)),
$$




$$
f(t) \otimes(g(t) * h(t))=(h(t) \otimes f(t)) \otimes g(t) .
$$

The following relations can also be obtained in a straightforward way:

$$
\begin{aligned}
& (f(t) \otimes g(t)) \otimes h(t)=(h(t) \otimes g(t)) \otimes f(t), \\
& f(t) \otimes(g(t) \otimes h(t))=g(t) \otimes(f(t) \otimes h(t)) .
\end{aligned}
$$

\section{APPENDIX C}

The frequency response of a compressed backscattering signal can be obtained by taking the Fourier transform of Eq. (15)

$$
Y(f)=\frac{k_{c}}{r^{2}} F_{\mathrm{bs}}(f) R_{s s}(f) e^{-i \omega t_{0}}+k_{c} R_{s n}(f),
$$

where $\quad F_{\text {bs }}(f), \quad R_{s s}(f)=S_{0}^{*}(f) S_{0}(f), \quad$ and $\quad R_{s n}(f)$ $=S_{0}^{*}(f) N(f)$ are the Fourier transforms of the signal $f_{\text {bs }}(t)$, autocorrelation $r_{s s}(t)$, and cross correlation $r_{s n}(t)$, respectively. $S_{0}(f)$ and $N(f)$ are Fourier transforms of the transmit signal, $s_{0}(t)$, and noise, $n(t)$, respectively. The power spectrum of the backscattering is then obtained by multiplying $Y^{*}(f)$ on both sides of Eq. (C1) and normalizing by $\left(k_{c} / r^{2}\right)^{2} R_{s s}(f) R_{s s}^{*}(f)$ :

$$
\begin{aligned}
P(f) & =\frac{r^{4} Y(f) Y^{*}(f)}{k_{c}^{2} R_{s s}(f) R_{s s}^{*}(f)} \\
& =\frac{\left[F_{\mathrm{bs}}(f) R_{s s}(f) e^{-i \omega t_{0}}+r^{2} R_{s n}(f)\right]\left[F_{\mathrm{bs}}^{*}(f) R_{s s}^{*}(f) e^{i \omega t_{0}}+r^{2} R_{s n}^{*}(f)\right]}{R_{s s}(f) R_{s s}^{*}(f)} \\
& =F_{\mathrm{bs}}(f) F_{\mathrm{bs}}^{*}(f)+\frac{F_{\mathrm{bs}}(f) N^{*}(f) e^{-i \omega t_{0}}}{S_{0}^{*}(f)} r^{2}+\frac{F_{\mathrm{bs}}^{*}(f) N(f) e^{i \omega t_{0}}}{S_{0}(f)} r^{2}+\frac{r^{4} N(f) N^{*}(f)}{S_{0}(f) S_{0}^{*}(f)} \\
& =\left|F_{\mathrm{bs}}(f)\right|^{2}+2 r^{2} \frac{\operatorname{Re}\left\{F_{\mathrm{bs}}(f) S_{0}(f) N^{*}(f) e^{-i \omega t_{0}}\right\}}{S_{0}(f) S_{0}^{*}(f)}+\frac{\left|r^{2} N(f)\right|^{2}}{\left|S_{0}(f)\right|^{2}} .
\end{aligned}
$$

The last expression in Eq. (C2) can be proven to be equivalent to that obtained by applying a standard spectral analysis to the original (unprocessed) time series. This result indicates that directly taking a Fourier transform of the CP output cannot improve the SNR of the signal in the frequency domain compared with a standard spectral analysis.

\section{APPENDIX D}

To evaluate the influence of the tether and the superglue used with the gastropods in the shipboard scattering experiments, data were collected without the presence of any ani$\mathrm{mal}$ in the acoustic beam. Only the tether and a small drop of superglue attached to it were in the acoustic beam. It was observed that the scattering contribution from the superglue was much larger than that from the tether. Since superglue was used only for the gastropod scattering experiment, our analysis of assessing signal contamination is based on the data associated with the configuration for the gastropod experiment.

The primary arrivals in $\mathrm{CP}$ outputs for siphonophores and gastropods are always very strong and can be considered as reliable echoes from the animals. This observation is consistent with the result of Stanton et al. (1998a). In that analysis, it is found that within the usable frequency band, the target strength of the combination of the tether and superglue is at least $6 \mathrm{~dB}$ lower than those from the gastropods used in the experiment which implies the SNR of the received scattering signal is always $6 \mathrm{~dB}$ higher than the background noise (tether, microstructure and other noise).
However, since the secondary arrivals are usually much smaller than the primary arrivals (except for shrimplike animals), a crucial concern to our CP analysis is that whether the secondary arrivals are real and reliable or whether they are contaminated by the presence of the tether, superglue (when it was used), and the microstructure, or even an artifact of PC processing.

One data set that involves only the tether and a small drop of superglue attached to the tether is used to evaluate how these factors affect our CP analysis. The data were acquired after a gastropod was carefully removed from the water (superglue was not visible on the removed animal). The transmitted signal was a chirp sweeping from $300 \mathrm{kHz}$ to $700 \mathrm{kHz}$ over a $200-\mu$ s period as shown in Fig. 2(e). To simulate a worst case, random noise with a constant SNR of

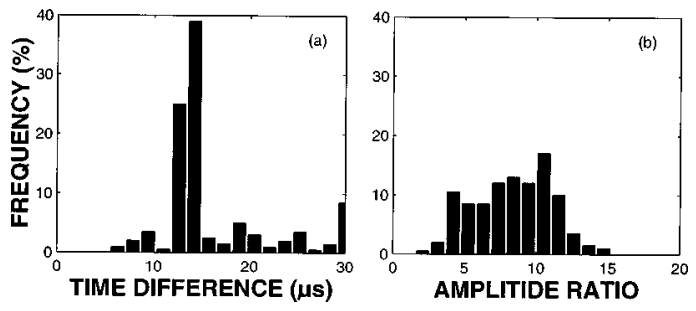

FIG. D1. Study of the influence of the superglue, the tether, microstructure, and artifacts of PC processing on the CP output. The histograms are obtained from the CP outputs from 200 pings. Random noise is added in such a way that the SNR of each ping is kept constant at $6 \mathrm{~dB}$. The signal here refers to the largest echo of the reverberation time series when no animal is attached to the tether. The transmit signal is a chirp signal shown in Fig. 2(e). 
$6 \mathrm{~dB}$ is added to each ping. In this case, the signal is the reverberation from the tether, microstructure, and superglue. The "replicate" of the signal used in the PC processing is the received signal in calibration mode shown in Fig. 2(e).

The statistics of the CP output of 200 pings of reverberation only (no animal) is shown in Fig. D1, where the time difference between primary and secondary arrivals and amplitude ratio of primary to secondary arrival are given in Fig. D1(a) and (b), respectively. The secondary arrivals are determined by selecting the second largest peak of the CP time series output regardless of whether the peak corresponds to the pre- or post-arrival. The time difference and the amplitude ratio shown in Fig. D1 are centered at $14 \mu \mathrm{s}$ and 9-10, respectively. From Fig. 2(f) (calibration mode), the time difference between the primary and the secondary arrivals is found to be $13.5 \mu \mathrm{s}$, and the amplitude ratio of the two arrivals is 11. Since the signal in Fig. 2(e) is the direct arrival from the bistatic calibration (where the tether and superglue are absent) and has a very high SNR, the influence of microstructure is weak and can be readily ignored. Thus it is reasonable to assert that the secondary arrivals in Fig. 2(f) stem from edges of the time series and are essentially (sidelobe) artifacts of the PC process as mentioned in Sec. I. Comparison of Fig. D1 and Fig. 2(f) reveals that the inclusion of tether and superglue as well as microstructure does not have a significant effect on the $\mathrm{CP}$ output (both time difference and amplitude ratio remain the same). We conclude that the error of our CP analysis is dominated by the artifact of the $\mathrm{PC}$ and the influence of the tether and the superglue on the $\mathrm{CP}$ output is not significant.

Abramowitz, M., and Stegun, I. A. (1965). Handbook of Mathematical Functions (Dover, New York).

Bracewell, R. N. (1986). The Fourier Transform and Its Applications (McGraw-Hill, New York).

Chu, D., Stanton, T. K., and Wiebe, P. H. (1992). "Frequency dependence of sound backscattering from live individual zooplankton," ICES J. Mar. Sci. 49, 97-106.

Chu, D., Foote, K. G., and Stanton, T. K. (1993). "Further analysis of target strength measurements of Antarctic krill at $38 \mathrm{kHz}$ and $120 \mathrm{kHz}$ : Comparison with deformed cylinder model and inference of orientation distribution,", J. Acoust. Soc. Am. 93, 2985-2988.

Clay, C. S. (1987). "Optimum time domain signal transmission and source location in a waveguide,'” J. Acoust. Soc. Am. 81, 660-664.

Clay, C. S., and Medwin, H. (1977). Acoustical Oceanography: Principles and Applications (Wiley-Interscience, New York).

Cook, C. E., and Bernfeld, M. (1967). Sonar Signals (Academic, New York).

Ehrenbreg, J. E., Ewart, T. E., and Morris, R. D. (1978). "Signal processing techniques for resolving individual pulses in a multipath signal," J. Acoust. Soc. Am. 63, 1861-1865.

Holliday, D. V., Pieper, R. E., and Kleppel, G. S. (1989). "Determination of zooplankton size and distribution with multi-frequency acoustic technology,' Journal du Conseil International pour L'Exploration de la Mer 46, $52-61$.

Holliday, D. V., and Pieper, R. E. (1995). "Bioacoustical oceanography at high frequencies," ICES J. Mar. Sci. 52, 279-296.

Parvulescu, A. (1961). "Signal detection in a multipath medium by M.E.S.S. processing,"' J. Acoust. Soc. Am. 33, 1674

Price, R. (1956). "Optimum detection of random signals in noise, with application to scatter-multipath communication,' IRE Trans. Inf. Theory IT-2, 125-135.

Robinson, E. A. (1980). Geophysical Signal Analysis (Prentice-Hall, Englewood Cliffs, NJ)

Siebert, W. Mc. (1956). "A radar detection philosophy," IRE Trans. Inf. Theory IT-2, 204-221.

Stanton, T. K., Chu, D., and Wiebe, P. H. (1998a). "Sound scattering by several zooplankton groups. I. Experimental determination of dominant scattering mechanisms,"' J. Acoust. Soc. Am. 103, 225-235.

Stanton, T. K., Chu, D., and Wiebe, P. H. (1998b). "Sound scattering by several zooplankton groups. II. Scattering models," J. Acoust. Soc. Am. 103, 236-253.

Stanton, T. K., Clay, C. S., and Chu, D. (1993a). "Ray representation of sound scattering by weakly scattering deformed fluid cylinders: Simple physics and application to zooplankton," J. Acoust. Soc. Am. 94, 34543462.

Stanton, T. K., Chu, D., Wiebe, P. H., and Clay, C. S. (1993b). “Average echoes from randomly oriented random-length finite cylinders: Zooplankton models,"' J. Acoust. Soc. Am. 94, 3463-3472.

Stanton, T. K., Wiebe, P. H, Chu, D., and Goodman, L. (1994a). “Acoustic characterization and discrimination of marine zooplankton and turbulence," ICES J. Mar. Sci. 51, 469-479.

Stanton, T. K., Wiebe, P. H., Chu, D., Benfield, M. C., Scanlon, L., Martin, L., and Eastwood, R. L. (1994b). "On acoustic estimates of zooplankton biomass," ICES J. Mar. Sci. 51, 505-512.

Thorne, P. D., Brudner, T. J., and Water, K. R. (1994). "Time-domain and frequency-domain analysis of acoustic scattering by spheres," J. Acoust. Soc. Am. 95, 2478-2486.

Thorne, P. D., Water, K. R., and Brudner, T. J. (1995). "Acoustic measurements of scattering by objects of irregular shape," J. Acoust. Soc. Am. 97, 242-251.

Turin, G. L. (1960). "An introduction to matched filters," IRE Trans. Inf. Theory IT-6, 311-329.

Van Trees, H. L. (1968). Detection, Estimation, and Modulation Theory (Wiley, New York), Pt. III.

Van Vleck, J. H., and Middleton, D. (1946). "A theoretical comparison of the visual, aural, and meter reception of pulsed signals in the presence of noise,' J. Appl. Phys. 17, 940-971.

Whalen, A. D. (1971). Detection of Signals in Noise (Academic, New York).

Winder, A., and Loda, C. J. (1981). Space-time Information Processing (Peninsula, Los Altos, CA), pp. 153-156.

Zhang, L. G., Sun, N. H., and Marston, P. L. (1992). "Midfrequency enhancement of the backscattering of tone bursts by thin elastic shells," $\mathrm{J}$. Acoust. Soc. Am. 91, 1862-1874. 